\title{
Palladium-Catalyzed Garbonyl Allylation Reactions Using Tin Chloride: A Mini-Review
}

\author{
In honor of Prof. Yoshiro Masuyama on his retirement celebration from Sophia University, \\ November 2017.
}

\author{
Mikael John A. Baltazar ${ }^{1}$, Roy Kristian C. Yanela ${ }^{1}$, Armando M. Guidote Jr. ${ }^{{ }^{*}}$, Noriyuki Suzuki ${ }^{*}$ \\ ${ }^{1}$ Department of Chemistry, School of Science \& Engineering, Loyola Schools, Ateneo de Manila University, \\ Quezon City 1108 \\ ${ }^{2}$ Department of Materials and Life Sciences, Faculty of Science and Technology, Sophia University, Tokyo, \\ Japan
}

\begin{abstract}
The treatment of allylic alcohols as synthons of carbanions for carbonyl allylation reactions in the presence of a Pd- $\mathrm{SnCl}_{2}$ system has been one of the most interesting and most useful developments demonstrated by Yoshiro Masuyama and co-workers in the field of organic synthesis. The reaction makes use of palladium as an effective catalyst and tin (II) chloride as a low-valent reducing agent which also effectively transforms the allylic group to a nucleophilic group. The organic, as well as organometallic, chemistry of how the transformations occur and how the metals take part in the reaction is of great interest. These could help allow for better optimization of reagents and solvents, for better control of the extent of reaction or yield of desired product, and for possible applications in other reaction systems. This review will focus primarily on the work of Yoshiro Masuyama and various co-workers on carbonyl allylation reactions making use of a $\mathrm{Pd}-\mathrm{SnCl}_{2}$ system or substitutes thereof.
\end{abstract}

Keywords: palladium catalysis; tin chloride reduction; allylic alcohols; organometallic chemistry

\section{INTRODUCTION}

$\pi$-Allylpalladium complexes have been generally applied as allylic cations in organic syntheses, using a variety of allylic derivatives such as allylic esters and halides. In this review, however, the focus will be on the use of an umpolung of metal-allyl complexes, i.e. treating the allylic species as the nucleophilic species instead of electrophile (Masuyama, 1994). As early as 1987, Yoshiro Masuyama and co-workers have begun investigating chemical couplings between allylic compounds and carbonyls. They explored the use of allylic acetates, a zinc-based reductant and a palladium catalyst yielding, however, low diastereoselectivity and long reaction times, extending up to 6 days (Masuyama, Kinugawa, \& Kurusu, 1987). Furthermore, the yield of allylic alcohol product was said to be quite low, especially in using only two equivalents of acetate, due to the competing homocoupling reaction between the allylic acetates (Sasaoka et al., 1985). These concerns were, however, circumvented upon change of solvent, from dioxane to 1,3-dimethylimidazolidin2-one (DMI), and of the metal reductant, from zinc to tin $\left(\right.$ as $\left.\mathrm{SnCl}_{2}\right)$. Notably, these changes prevented homocoupling of the acetates and reduced the reaction times from 4-6 days to only around 20-60 hours (Masuyama, Otake, \& Kurusu, 1988). In a later paper of Masuyama and co-workers of the same year (Masuyama, Hayashi, Takahara, \& Kurusu, 
1988), they have proposed the use of allylic alcohols instead of allylic acetates as synthons of allylic carbanions. Notable characteristics of the reaction were that (1) the molar amount of $\mathrm{SnCl}_{2}$ must be twice that of the allyl alcohol, (2) reactions with ketones were more favorable compared to the use of $\mathrm{Zn}$ and allylic acetates, and (3) better regioselectivity was also observed, as will be discussed in this paper. This reaction system (Scheme I) has been utilized by Masuyama and co-workers (1988) for quite a number of studies and will be the focus of this review.

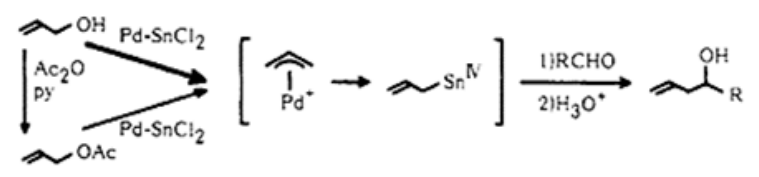

Scheme I. Carbonyl allylation using either allyl alcohol or allyl acetate in the presence of $\mathrm{Pd}-\mathrm{SnCl} \mathrm{l}_{2}$. [Reprinted with permission from $Y$. Masuyama et al. (1988). J. Am. Chem. Soc. 110, 4473-4474. Copyright (C) 1988, The Royal Society of Chemistry]

\section{ORGANOMETALLICS: PALLADIUM CATALYSIS}

Prior to discussing the reaction of interest, some background must be given on the reagents used. For this section, we will focus on the primary metalbased catalyst used for this system, namely palladium complexes. Matthew Hartings in his review (2012) has stated that one "would be forgiven if [one] thought the most important element in an organic transformation was carbon. [He] argues that, for just over half a century in many of chemistry's most renowned organic reactions, it has actually been palladium." As bold as the claim may seem, it does have its merit in how a number of organic processes

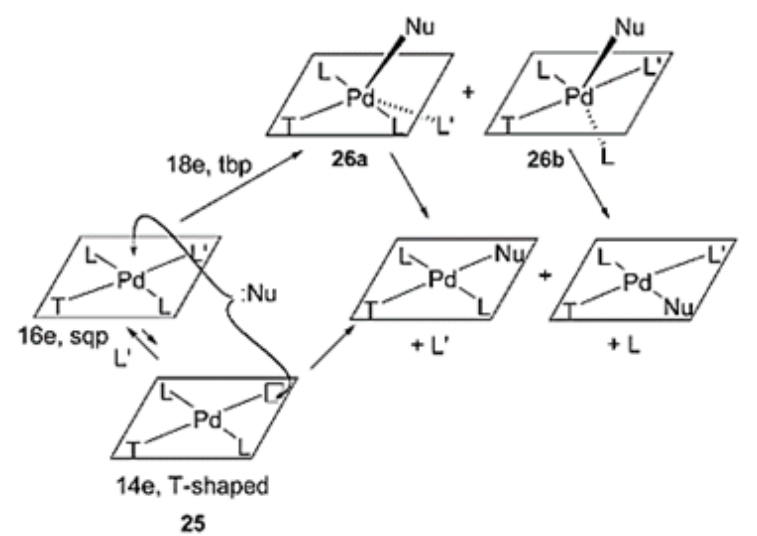

Scheme II. Proposed reaction pathways for transmetallation. [Reprinted with permission from $P$. Espinet \& A. Echavarren (2004). Angew. Chem. Int. Ed. 43, 4704-4734. Copyright (C) 2004, John Wiley \&. Sons, Inc.] known today, especially that of carbon-carbon couplings, make use of palladium catalysts. A few reactions to name would be the Heck reaction, the Suzuki coupling reaction, and the Tsuji-Trost allylation (Caspi, 2008).

In the reaction of interest, palladium is involved in the formation of a $\pi$-allylpalladium complex that gears the substrate for addition of the tin chloride reagent. Several other reactions use a similar function for the metal. To get a better understanding of how this occurs, a review of some typical catalytic cycle reactions will first be discussed, especially considering palladium catalysis. These key reactions are 1) transmetallation, 2) reductive elimination, and 3) oxidative addition, wherein the last reaction appears to be intimately involved in the transformation of interest, as will be discussed under oxidative addition.

Transmetallation. By definition, transmetallation (alternative spelling: transmetalation) involves the transfer of an alkyl group or other organic ligand from one metal, e.g. $\mathrm{Zn}, \mathrm{Mg}$ or $\mathrm{Li}$, to another (Crabtree, 2005). In a paper discussing the mechanism of the Stille reaction (Espinet \& Echavarren, 2004), the authors described the transmetallation process with palladium as occurring in the +2 oxidation state of the metal, and starts from a 16-electron tetracoordinated square planar species. Though the steps of transmetallation are not very well understood, they proposed that it could either occur by a dissociative or associative pathway (Scheme II). As can be seen, the dissociative pathway involves a 14-electron T-shaped intermediate, while the associative pathway goes through an 18-electron bipyramidal intermediate complex. The authors also note that solvent molecules may play a role in

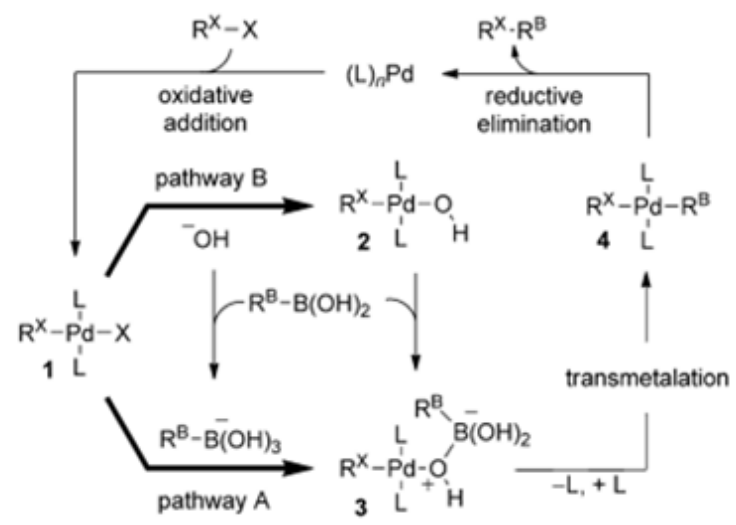

Scheme III. Simplified generic reaction scheme for Suzuki-Miyaura coupling of boronic acid $\left(\mathrm{RBB}(\mathrm{OH})_{2}\right)$ with organo-halide $(R X X)$. $L=$ ligand or solvent; $X=$ halide; only trans isomers illustrated. [Reprinted with permission from A. Lennox \& G. Lloyd-Jones (2013). Angew. Chem. Int. Ed. 52, 7362-7370. Copyright (C) 2013, John Wiley \& Sons, Inc.] 
directing substitution, such as by initial coordination of solvent to the metal, and thus affect the reaction kinetics. Transmetallation processes are also typically found midway in the catalytic cycle, such as in the Suzuki-Miyaura coupling reaction (Scheme III; Carrow \& Hartwig, 2011; Lennox \& LloydJones, 2013). Transmetallation between palladium and other metal complexes has found numerous applications for synthetic routes, especially for industrial applications (Albéniz, Espinet \& Pérezmateo, 2010; Lei, Srivastava \& Zhang, 2002; Miyaura, 2002).

Reductive Elimination. Reductive elimination, on the other hand, involves, as the name implies, reduction of the metal of interest's oxidation state by two units and the formation of a sigma $(\sigma)$ bond between two ligands. Typically, in catalytic reactions, it is the last step in the catalytic cycle, regenerating the initial form of the metal catalyst and yielding the desired product. The reduced form of the metal must then be stable enough to re-enter the catalytic cycle. In light of the principle of microscopic reversibility, it can be assumed that the mechanisms by which reductive elimination occurs must be similar to that of oxidative addition (only in reverse), some of whose proposed reaction schemes will be discussed under oxidative addition (Crabtree, 2005).

Many factors can affect the favorability and rate of elimination, such as the nature of the ligand (e.g. stability of R-R' vs. R-M-R'), the metal used and what other reactions the elimination can be coupled to. It is also quite common for the thermodynamic and kinetic characteristics of an organometallic reaction to differ significantly. In a study of aryl halides, for example, using palladium catalyst (as ( $\mathrm{t}-\mathrm{Bu})_{3} \mathrm{P}-$ $\mathrm{PdArX}$ ), equilibrium studies showed that reductive elimination of aryl chlorides are thermodynamically more favorable than aryl bromides and iodides, yet the aryl chloride formed more slowly compared to the bromide and iodide. The thermodynamic data revealed the dominant effects of aryl-halide bond strengths, while for the kinetic case, transition state energies are more contributing (Roy \& Hartwig, 2003). Interestingly, acceleration of reductive elimination was also observed when coupled to the oxidative addition of allyl chloride to the palladium metal (Kurosawa, Emoto \& Kawasaki, 1988).

Oxidative Addition. In oxidative addition, a molecule, R-R', is split and coordinates to the metal as M-R and M-R'. Since R and R' are X-type ligands, they each reduce electron density on the metal and increase its oxidation state by a total of two units, along with electron count and coordination number. The latter two mentioned changes imply the need for a vacant 2 -electron site on the metal. This thus means that the metal complex must be a 16-electron complex or, if starting as an 18-electron complex, must supply a vacant site by ligand dissociation (Crabtree, 2005). As with many other transition metals, palladium is often invoked, usually as $\operatorname{Pd}(0)$, to initiate the catalytic cycle via oxidative addition. Numerous coupling reactions make use of palladium for this (Caspi, 2008), e.g. Suzuki-Miyaura coupling (Scheme III).

Oxidative additions are said to bevery mechanistically diverse. For example, the reaction is said to possibly occur by a three-center concerted mechanism, wherein the incoming ligand (R-R') first associates with the metal through its $\sigma$ bond, forming a $\sigma$ complex, then undergoes R-R' bond breaking due to back donation of the metal to its antibonding orbital (Scheme IV).

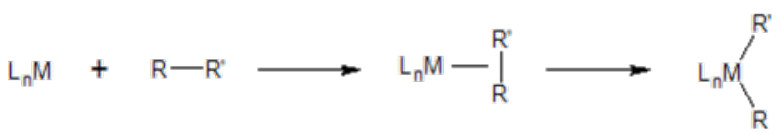

Scheme IV. General reaction pathway for oxidative addition of ligand $(R-R$ ') to metal $(M)$. [Adapted from R. Crabtree. Copyright (C) 2005, John Wiley \& Sons, Inc.]

A non-concerted $S_{N} 2$-like mechanism has also been proposed. This is said to be more common for polarized substrates such as alkyl halides, wherein the metal electrons (from LnM) directly attack the R-R' $\sigma^{*}$ orbital (Scheme V). This reaction is highly reminiscent of organic $S_{N} 2$ reactions, with stereochemical inversions at the carbon atoms of substituted halides being commonly observed (Crabtree, 2005).

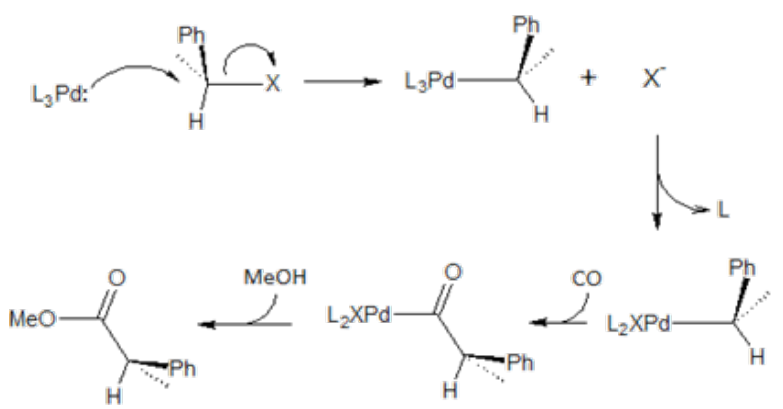

Scheme $V$. Non-concerted $S_{N} 2$-like mechanism of oxidative addition. Metal electrons attack the ligand $\sigma^{*}$ orbitals. [Adapted from R. Crabtree. Copyright (C) 2005, John Wiley \& Sons, Inc.]

Other possible routes include radical and ionic mechanisms. The former typically involves single electron transfer (SET) mechanisms, as in the Grignard or Barbier reactions, while the latter involves initially ionized ligands (e.g. $\mathrm{L}_{\mathrm{n}} \mathrm{M}+\mathrm{H}^{+}+\mathrm{Cl}^{-} \rightarrow \mathrm{L}_{\mathrm{n}} \mathrm{HMCl}$ ). Such reaction schemes were deduced by spectroscopic studies (e.g. NMR kinetic studies, electron spin resonance) or by isotopic labeling (Crabtree, 2005). 
As previously stated, oxidative additions are typically involved in the beginning of catalytic cycles, adding the reacting substrate to the metal to mediate reaction with another reagent. For the case of the carbonyl allylation in this review, oxidative addition of the allyl to the palladium (0) catalyst involves dissociation of a leaving group (e.g. $\mathrm{OH}^{-}$ or acetate) and coordination of the allyl to the metal via a 3-carbon coordination, effectively also oxidizing the metal to its +2 oxidation state (Scheme I). Palladium is said to form allylic complexes easily in the +2 oxidation state as it is easy to prepare and handle and typically acts as an intermediate for various palladium-catalyzed coupling reactions. The oxidative addition of palladium to allylic chlorides and alcohols have been described as early as the 1960 s with the latter being promoted in the presence of acid, due to protonation of the $-\mathrm{OH}$ group (Maitlis, 1971). The aforementioned leaving group (e.g. $\mathrm{OH}^{-}$or acetate), on the other hand, is typically described to act as the counter ion for the Pd-allyl complex, generated by what is described as reversible oxidative addition (Agenet et al., 2002; Amatore et al., 2000; Jutand, 2004). Interestingly, a study using iridium as transition metal has regarded the $\mathrm{S}_{\mathrm{N}}$ 2-like mechanism as the most probable route for $\pi$-allylmetal complex formation. In this model, the metal is described to attack the leaving group (e.g. halide)-bonded carbon atom, as the leaving group departs, allowing the formation of the complex (Pearson \& Poulos, 1979). The characteristics of this organometallic reaction will be discussed later.

\section{TIN (II) CHLORIDE CHEMISTRY}

Aside from its +4 oxidation state, tin has shown increasing importance and applicability in its +2 oxidation state (Miessler, Fischer \& Tarr, 2014). In this form, tin (II) chloride, known as stannous chloride, is regarded as a highly versatile reagent for organic syntheses. For this type of dihalide, tin serves as a low valent metal which enables the activation of substrates rich in electrons. As such, it can serve as Lewis acid, being able to accept electrons in its $d$ orbitals.

In many circumstances, $\mathrm{SnCl}_{2}$ serves as a mediator in many metal-catalyzed reactions. This holds true in the subject of interest as $\mathrm{SnCl}_{2}$ can impart distortions on the electrons of the $\pi$-allylpalladium complex. Upon exchange with low valent metals, the so-formed allyl metal species can now function as a nucleophile, instead of the usual electrophile, hence an umpolung. This ability of the low valent metal permits transmetallation with another metal, consequently allowing the allylation of carbonyl compounds (Frost, Howarth \& Williams, 1992).

\section{Pd-SnCl ${ }_{2}$ MEDIATED CARBONYL ALLYLATION REACTION}

Yoshiro Masuyama and various co-workers have published numerous papers with regards to the application of the $\mathrm{Pd}-\mathrm{SnCl}_{2}$ system in carbonyl allylation reactions/syntheses. In this section, some of these papers will be discussed with an emphasis on the mechanistic insights gained for the reaction of interest. Work by other authors relevant to the subject at hand will also be included in subsequent sections.

In developing the allylation reaction system, many factors were considered. The choice of metal reagent to use was by far the most important consideration. In light of this, Masuyama and co-workers had many questions and concerns in mind. For example, was charge reversal of the $\pi$-allylpalladium complex with the chosen metal reagent possible? Single-electron transfer reactions should also be discouraged by the system, else homocoupling reactions would be rampant. It was also highly preferable for the metal reagent to regioselectively attack the $\pi$-allylpalladium complex for practical synthetic applications. And finally, the nucleophilic addition of the allylmetal to the carbonyl compound preferably must occur in the same pot (Masuyama, 1994).

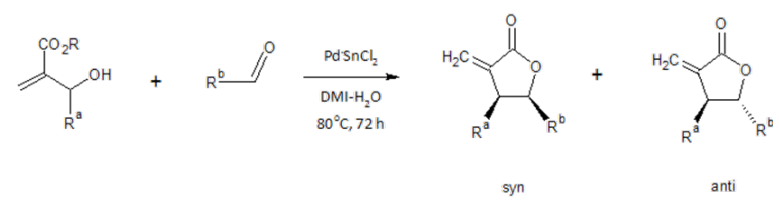

Scheme VI. Reaction system for the synthesis of $\alpha$-methylene- $\gamma$-butyrolactone from ethyl 2-(hydroxymethyl) acrylate with aldehyde in the presence of $\mathrm{Pd}-\mathrm{Sn} \mathrm{Cl}_{2}$. [Adapted from Y. Masuyama et al. (1991) Tetrahedron Letters. 32 (2), 225-228. Copyright (C) 1991, Elsevier]

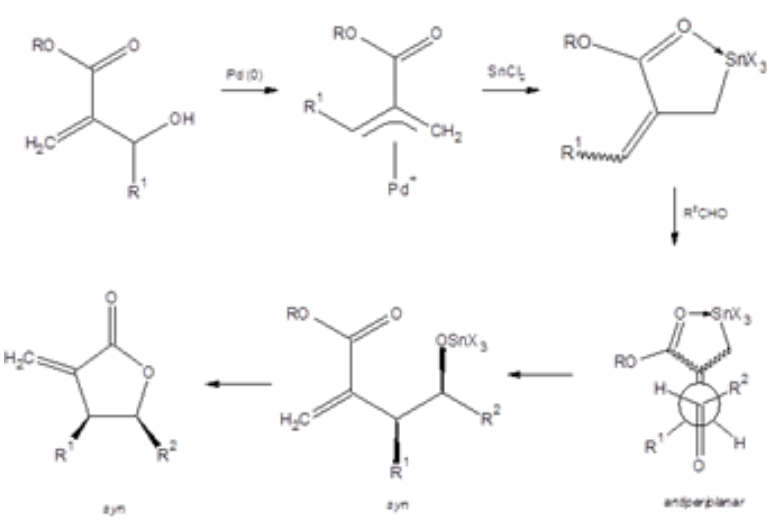

Scheme VII. Proposed carbonyl allylation pathway towards syn selection. [Adapted from Y. Masuyama et al. (1991) Tetrahedron Letters. 32 (2), 225-228. Copyright (C) 1991, Elsevier] 
Table 1. Addition reaction of 2-butenol (1) to benzaldehyde under various conditions [Reprinted with permission from Takahara et al. (1992) J. Am. Chem. 114, 2577-86. Copyright ()$_{1}$ 1992, American Chemical Society].

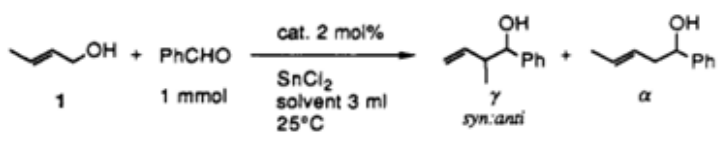

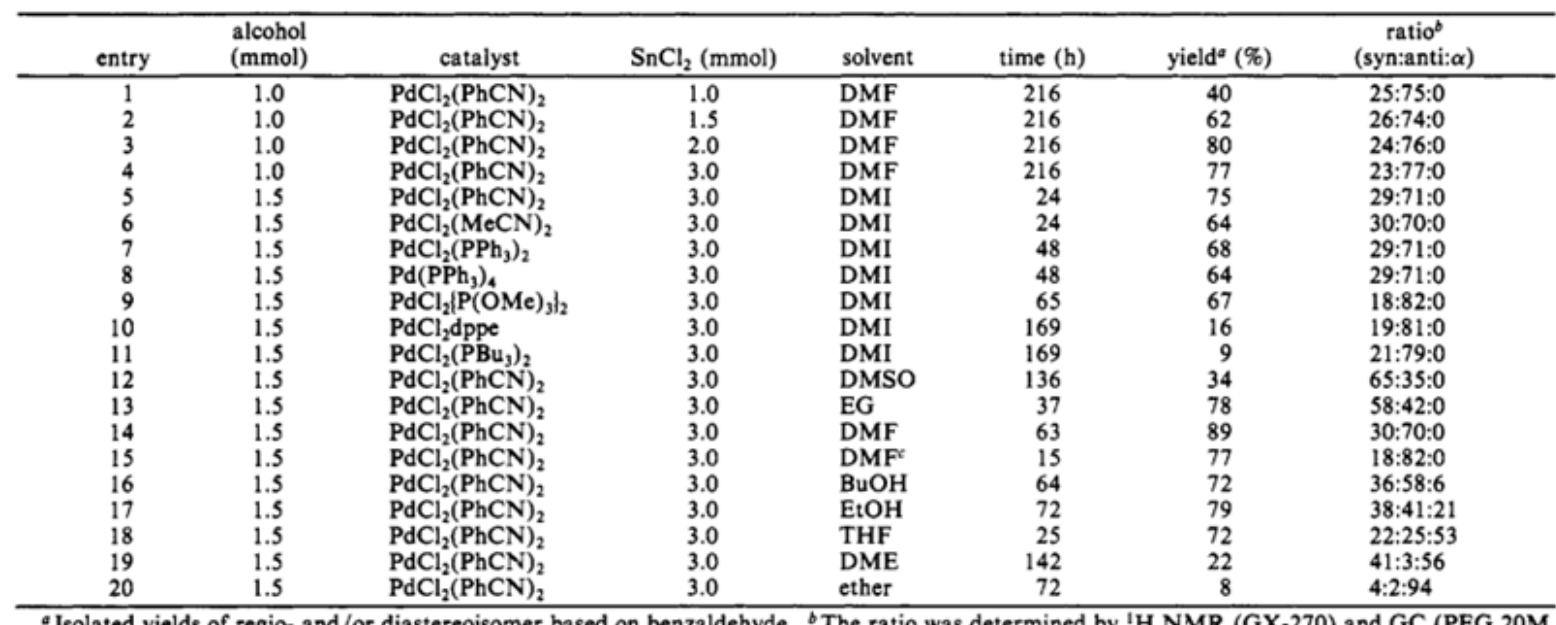

$0.25 \mathrm{~mm} \times 30 \mathrm{~m}) .{ }^{'} \mathrm{H}_{2} \mathrm{O}(1 \mathrm{~mL})$ was added.

Lactone Formation from Acrylic Ester with an Allylic Alcohol Group. In 1991, the preparation of $\alpha$-methylene- $\gamma$-butyrolactones from an ethyl 2-(hydroxymethyl)acrylate has been reported. This was a development from 2-(bromomethyl) acrylates which were said to be difficult to prepare and to store due to instability. The reaction system was described to involve a palladium catalyst in the form of $\mathrm{PdCl}_{2}(\mathrm{PhCN})_{2}$, a tin (II) chloride reductant and the primary reacting species, the aldehyde and 2-(hydroxymethyl) acrylate, in 1,3-dimethylimidazolidinone (DMI)$\mathrm{H}_{2} \mathrm{O}$ as solvent to give the butyrolactone product (Scheme VI). For most carbonyl compounds utilized, however, the yield played around values of $23-47 \%$. Investigation of diastereoselectivity, nevertheless, showed almost $100 \%$ syn selection (Masuyama, Nimura, \& Kurusu, 1991). Of greater interest in these reactions was the intermediates believed to have formed to yield the syn-selected product. As in Scheme I, the authors have shown the initial formation of a $\pi$-allylpalladium complex intermediate and subsequent attack of the tin species. Syn addition then proceeded through what is described as an acyclic antiperiplanar intermediate, suggesting the chelation of the ester oxygen group to the $\mathrm{Sn}$ (IV), effectively preventing the formation of a cyclic intermediate (Scheme VII).

Characteristics of Palladium-Catalyzed Carbonyl Allylation with $\mathrm{SnCl}_{2}$. In the succeeding year, Masuyama and co-workers submitted an investigative paper, trying to optimize the reagents, catalyst, solvent used and other parameters used for the allylation reaction. They also looked more closely into the selectivity and actual mechanism of the allylation reaction (Takahara, Masuyama \& Kurusu, 1992). They claimed that the use of tin (II) chloride as a reducing low-valent metal is more effective compared to using other such metals or salts. They have also regarded the use of allylic alcohol as highly preferable than allylic esters and halides since derivatization to the latter entails use of more resources. But aside from that, in their preliminary study, allylic alcohol reactivity towards palladium-catalyzed carbonyl allylation was found to be greater than allylic esters, and so usage of allylic alcohol in the investigation was deemed more useful.

Choice of Catalyst. The first table (Table 1) highlights several key insights from their investigation. For example, entries 5-11 show several forms or preparations of the palladium catalyst and reveal the $\mathrm{PdCl}_{2}(\mathrm{PhCN})_{2}$ and $\mathrm{PdCl}_{2}(\mathrm{MeCN})_{2}$ forms to be superior with regards to catalytic activity compared to the other tested complexes; the reaction time is seen to be greatly reduced. It is unclear, however, if the ligand effects are due to size or electronwithdrawal capacity. But the superiority of such complexes compared to other Pd complexes bearing phosphine ligands can be heavily attributed to their stability in air.

Effect of Choice of Solvent. The choice of solvent also observably affected the yield, regio- and stereoselectivity (Table 1, entries 12-20). Polar solvents (entries 12-17) tended to favor the $\gamma$-product, while less polar ones (entries 18-20) 
generally depressed the yield and favored $\alpha$ selection. The regioselectivity of the reactions in these solvents conforms to the known dielectric constants and Swain's parameters of solvent which says that higher regioselectivity (i.e. more $\gamma$-addition) is attainable in solvents with larger dielectric constants. So to speak, syn selection can be achieved by the use of ethylene glycol (EG) and dimethylsulfoxide (DMSO), while anti selection can be achieved using 1,3-Dimethyl2-imidazolidinone (DMI) and dimethylformamide (DMF). Another point of interest in the data is the comparison between the presence and absence of water in the same reaction system (Table 1, entries 14-15). The reaction time was, surprisingly, significantly reduced, from $63 \mathrm{~h}$ to just $15 \mathrm{~h}$ in the presence of water. Interestingly, other work involving tin-mediated carbonyl allylations have also reported tolerance and acceleration in the presence of water, even with acid, although it remains unclear how it occurs (Guimarães et al., 2007; Tan, Hou \& Liu, 2004). But kinetics aside, the presence of water in the reaction system has shown significant effects in regio- and stereoselectivity as well (Table 1, entries 14-15). An investigation on the regio- and diastereoselection of benzaldehyde with 2-(E/Z)butenol pointed out that higher proportions of water in the reaction system, as in the THF- $\mathrm{H}_{2} \mathrm{O}$ solvent system, generally favors $\gamma$-addition and the formation of the anti-isomer product. While the DMSO- $\mathrm{H}_{2} \mathrm{O}$ system permits non-diastereoselection, the presence of large amounts of water still allows anti-selection.

Mechanistic Aspects of the Reaction. Another key observation by Takahara and co-workers was that the allylation needed two equivalents of $\mathrm{SnCl}_{2}$ for significant yield (Table 1, entries 1-4). Due to this, two functions have been proposed for $\mathrm{SnCl}_{2}$ : (1) acting as a Lewis acid to aid elimination of the $-\mathrm{OH}$ group and (2) serving as the reducing agent for the $\pi$-allylpalladium complex.

The mechanism of action of the $\mathrm{SnCl}_{2}$ reagent in the carbonyl allylation reaction has been largely ambiguous, most especially with respect to the intermediate species that it forms. Takahara and co-workers inferred an allylic tin intermediate and carried out NMR spectroscopic studies to prove their claim. They compared the ${ }^{1} \mathrm{H}$ and ${ }^{13} \mathrm{C}$ NMR of the reaction product of 2-propenol (1 eq.) and $\mathrm{SnCl}_{2}$ (2 eq.) with 3-chloropropene (1 eq.; Scheme VIII, 3) and $\mathrm{SnCl}_{2}$ (2 eq.) with both systems in the presence of $\mathrm{PdCl}_{2}(\mathrm{PhCN})_{2}$ in DMF- $\mathrm{d}_{7}$ at $25^{\circ} \mathrm{C}$, and the results showed largely similar spectra for both cases (Takahara, Masuyama \& Kurusu, 1992). Comparison of the product spectra with literature (Denmark, Wilson T \& Wilson TM, 1988) also showed numerous similarities, thus allowing the inference of an allyl trichlorotin intermediate
(Scheme VIII, 5). From these findings, it thus appears that allyl- $\mathrm{SnCl}_{3}$ must be the actual allylating agent, attacking the carbonyl compound.

Many other studies, including other authors, assert that the allylation reaction can be best described as a Barbier-type allylation, which is essentially an in situ version of the well-known Grignard reaction involving either carbanion-type attack on the carbonyl or single electron transfer, SET (Wang, 2010). Masuyama, himself, has stated the Barbier-type nature of the reaction with tin (II) chloride (Masuyama, Takeuchi \& Kurusu, 2005; Masuyama, Saeki, Horiguchi \& Kurusu, 2001). Carbonyl allylation in an organic-aqueous solvent mediated by $\mathrm{SnCl}_{2}$ in the presence of copper as catalyst/activator has also been reported (Tan, Hou \& Liu, 2004). Of interest in the cited authors' work is their observation that water greatly accelerated the reaction and improved the yield compared to using only organic solvent, e.g. dichloromethane (DCM). From only around $50 \%$ yield, at a ratio of just 20:1 DCM-water, the yield went all the way to around $100 \%$, consistent with the observations of Takahara et al. (1992). Interestingly, the organometallic chemistry seems to take a different turn when another metal (aside from Pd) is used as catalyst. Rhodium has shown some promise as a catalyst, but, to date, iridium seems to have the highest output/yield out of the three (Pd, Rh and Ir) which likely relates to catalytic efficiency/turnover (Masuyama, Kaneko, \& Kurusu, 2004; Masuyama \& Marukawa, 2007). An investigation by Banerjee and Roy (2006) further explored the characteristics of the $\mathrm{SnCl}_{2}$-iridium-mediated allylation and found that no allyltin intermediate could be detected. Instead, a Grignard-type of insertion was proposed,

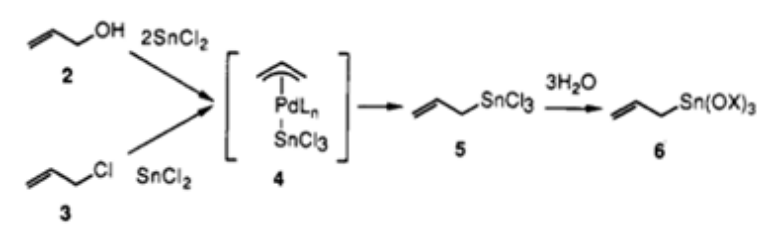

Scheme VIII. Proposed reaction pathway for 2-propenol (parallel to 3-chloropropene) to inferred intermediate 5 Reprinted with permission from Takahara et al. (1992) J. Am. Chem. 114, 2577-86. Copyright (C) 1992, American Chemical Society]

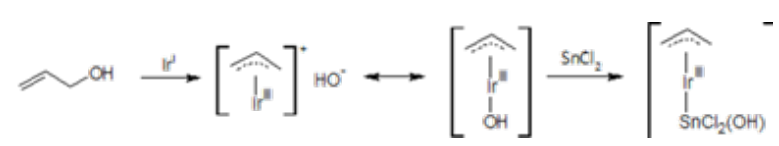

Scheme IX. Proposed reaction pathway intermediates for $\mathrm{Ir}-\mathrm{SnCl} \mathrm{Cl}_{2}$ mediated carbonyl allylation [Adapted from Banerjee \& Roy. (2006) Journal of Molecular Catalysis A. 246, 231-236. Copyright (C) 2006, Elsevier]. 
forming an Ir-Sn-X intermediate, where $\mathrm{X}$ is the leaving group (Scheme IX). This illustrates how much more complex the reaction of interest may be than previously thought. Further investigations are needed for a more conclusive picture of the reaction pathway, the role of transition metal catalysts, and the role of the metal salt reductant.

Chemoselection in Carbonyl Allylation. Another point of interest in the investigation of Takahara and co-workers (1992) was the chemoselectivity achieved by the reaction system. Though somewhat predictably, addition was selective towards aldehydes rather than ketones, with higher yields and lower reaction times needed for allylation of aldehydes. This was likely due to steric effects. With regards to reactivity of different allylic derivatives, the order of increasing leaving group reactivity was found to be: allylic acetate < allylic alcohol < allylic carbonate, similar to their preliminary study. Other studies support this finding with allylic alcohols and carbonates generally performing better than allylic phenoxides, phosphates and ethers. Lewis acid-assisted elimination of hydroxyl groups would explain the reactivity of allylic alcohols, while it has been reasoned that allylic carbonates can undergo decarboxylative allylations (Tsuiji, Shimizu, Minami \& Ohashi, 1982; Tsuji et al., 1985). Similar to the case of chemoselection, some other aspects of stereoselectivity, such as diastereoselection and asymmetric induction, were reported to be affected by bulky substituents on either or both the aldehyde and the allylic alcohol.

\section{FURTHER WORKS AND INVESTIGATIONS ON CARBONYL ALLYLATION}

Although the exact reaction mechanism has somewhat remained ambiguous in the case of the carbonyl allylation, further synthetic developments of the same reaction system or of similar nature have been reported by Masuyama's group. Discussed in this section are some of their other works that provide information on the potential applications and versatility of the $\mathrm{Pd}-\mathrm{SnCl}_{2}$ system in carbonyl allylation.

Carbonyl Allylation by Allylic Diols and Aliphatic Alkenes. Following the 1992 work by Takahara et al., an investigation on aldehyde allylation of 2-methylenepropane-1,3-diol was performed (Masuyama, Kagawa \& Kurusu, 1996). The transformation was accomplished using $\mathrm{PdCl}_{2}(\mathrm{PhCN})_{2}, \mathrm{SnCl}_{2}$, and DMF or THF (as solvent) at room temperature. With the main substrate bearing two identical allylic alcohol moieties, Masuyama and co-workers were able to synthesize
Table 2. Generic reaction of 2-methylenepropane1,3-diol with aldehydes and some of the obtained results [Reprinted with permission from Masuyama et al. (1996) Chemical Communications. 1585-1586. Copyright (c) 1996, The Royal Society of Chemistry].

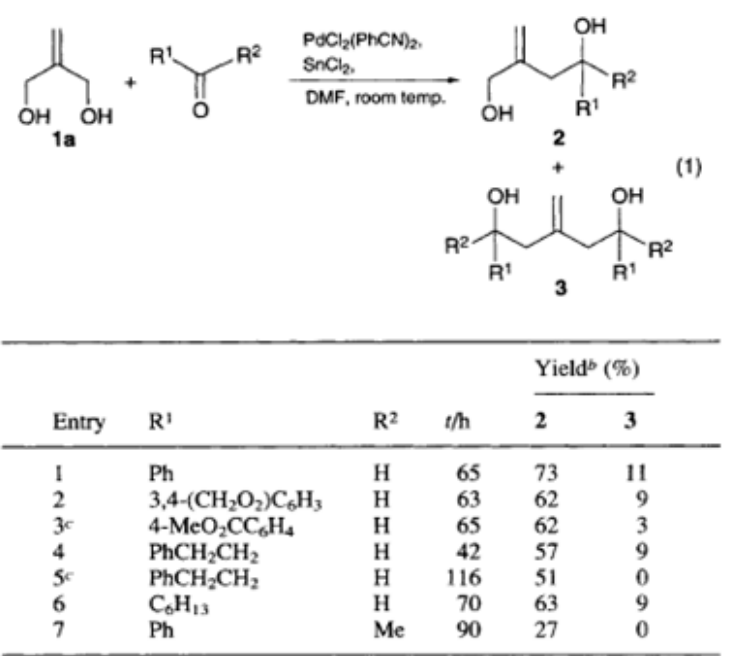

Monoallylation of aldehydes (1 mmol) by 1 a $(2 \mathrm{mmol})$ was carried out with $\mathrm{PdCl}_{2}(\mathrm{PhCN})_{2}(0.02 \mathrm{mmol})$ and $\mathrm{SnCl}_{2}(2 \mathrm{mmol})$ at room temp. in DMF $(3 \mathrm{ml})$. ${ }^{b}$ Isolated yields. $<$ THF was used as a solvent.

Table 3. Generic reaction of isoprene with aldehydes and some of the obtained results [Reprinted with permission from Masuyama et al. (1994) J. Chem. Soc., Chem. Commun. 14511452. Copyright (c) 1994, The Royal Society of Chemistry].

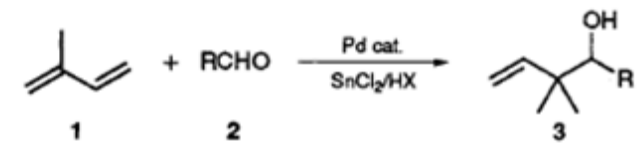

\begin{tabular}{llll}
\hline Entry & $\begin{array}{l}\text { Aldehyde } \\
\mathrm{R}\end{array}$ & Catalyst & $\begin{array}{l}\text { Yicld } b \\
(\%)\end{array}$ \\
\hline 1 & $4-\mathrm{NCC}_{6} \mathrm{H}_{4}$ & $\mathrm{Pd}(\mathrm{OAc})_{2}$ & 61 \\
2 & $4-\mathrm{MeO}_{2} \mathrm{CC}_{6} \mathrm{H}_{4}$ & $\mathrm{Pd}\left(\mathrm{PPh}_{3}\right)_{4}$ & 82 \\
3 & $2-\mathrm{BrC}_{6} \mathrm{H}_{4}$ & $\mathrm{Pd}\left(\mathrm{PPh}_{3}\right)_{4}$ & 57 \\
4 & $4-\mathrm{ClC}_{6} \mathrm{H}_{4}$ & $\mathrm{Pd}\left(\mathrm{OAc}_{2}\right.$ & 58 \\
5 & $4-\mathrm{MeOC}_{6} \mathrm{H}_{4}$ & $\mathrm{Pd}(\mathrm{OAc})_{2}$ & 46 \\
6 & $\mathrm{PhCH}_{2} \mathrm{CH}_{2}$ & $\mathrm{Pd}\left(\mathrm{OAC}_{2}\right.$ & 31 \\
7 & $n-\mathrm{C}_{10} \mathrm{H}_{21}$ & $\mathrm{Pd}\left(\mathrm{PPh}_{3}\right)_{4}$ & $35^{d}$ \\
8 & $\mathrm{CH}_{2}=\mathrm{CH}_{2}\left(\mathrm{CH}_{2}\right)_{8}$ & $\mathrm{Pd}\left(\mathrm{PPh}_{3}\right)_{4}$ & $32^{d}$ \\
9 & $c-\mathrm{C}_{6} \mathrm{H}_{11}$ & $\mathrm{Pd}\left(\mathrm{PPh}_{3}\right)_{4}$ & $17^{d}$ \\
\hline
\end{tabular}

a The allylation of aldehyde $(1 \mathrm{mmol})$ by isoprene $(5 \mathrm{mmol})$ was carricd out with Pd catalyst $(0.02 \mathrm{mmol}), \mathrm{PPh}_{3}(0.08 \mathrm{mmol})$, and $\mathrm{SnCl}_{2}$ $(2 \mathrm{mmol})$ at $40^{\circ} \mathrm{C}$ for $24 \mathrm{~h}$ in $\mathrm{H}_{2} \mathrm{O}(1 \mathrm{ml})$ and $\mathrm{AcOH}(2 \mathrm{ml}) .{ }^{b}$ Isolated yiclds. " The reaction was carried out at $80^{\circ} \mathrm{C}$ for $2 \mathrm{~h} .{ }^{d}$ The reaction was carried out with $\mathrm{PPh}_{3}(0.04 \mathrm{mmol})$ at $50{ }^{\circ} \mathrm{C}$. The regioisomers. 1-substituted 4-methylpent-3-en-1-ols, were obtained in $6-11 \%$ yields.

the monoallylated product under DMF. Using THF, however, depressed the yield of the monoallylated product but increased the reaction selectivity. As for the case of 1,3-dichloro-2-methylenepropane, reacting it with aldehyde under the same conditions 
led to the formation of a diallylated product (not shown on Table 2). Upon closer investigation, this work was able to demonstrate the selectivity of the process towards monoallylation.

Carbonyl allylation by simple aliphatic alkenes has also been a subject of interest by Masuyama's group. In 1994, Masuyama and co-workers were able to explore this using palladium as catalyst, $\mathrm{SnCl}_{2}$, and an $\mathrm{H}_{2} \mathrm{O}-\mathrm{AcOH}$ solvent system. The transformation was done under $50-80{ }^{\circ} \mathrm{C}$ temperature, depending on the aldehyde compound, for a duration of at most $24 \mathrm{~h}$. The yield was observed at around 17$82 \%$ (Table 3). The lower yields can be attributed to the use of a bulky aldehyde, as less bulky and aromatic aldehydes bearing electron-donating and electron-withdrawing groups produced more moderate yields.

Stereoselection in Carbonyl Allylation. In 1998, another diastereoselectivity investigation was performed (Masuyama,Ito \& Kurusu, 1998). This method utilized mixed (E)- and (Z)-1,3dichloropropene with $\mathrm{SnI}_{2}-\mathrm{Bu}_{4} \mathrm{NI}$ and $\mathrm{SnCl}_{2}$ to produce syn and anti 1-substituted 2-chlorobut3-en-1-ol, respectively. Reaction conditions were particularly mild but require long reaction periods (62-97 hrs). Although the corresponding yields were generally moderate (24-78\%), selectivity was found to be excellent (Table 4).
Table 4. Generic reaction of $(E)$ - and $(Z)-1,3-$ dichloropropene with aldehydes and some of the obtained results [Reprinted with permission from Masuyama et al. (1998) Chemical Communications. 315-316. Copyright (c) 1998, The Royal Society of Chemistry].

\begin{tabular}{|c|c|c|c|c|}
\hline $\mathrm{Cl}_{1}^{\mathrm{Cl}} \frac{\mathrm{A}}{\mathrm{B}}$ & $\begin{array}{r}\text { A: (1) } \mathrm{Pd} / \mathrm{SnCl}_{2}, \mathrm{DM} \\
\text { room temp., } \\
\text { (2) RCHO, } 0^{\circ} \mathrm{C} \\
\text { B: (1) } \mathrm{Pd} / \mathrm{Snl}, \mathrm{DMM}- \\
\text { room temp., } \\
\text { (3) } \mathrm{TBAA-Nal,} \mathrm{roo} \\
\text { (3) RCHO }\end{array}$ & $\begin{array}{l}\text { omp. } 3 \mathrm{~h} \\
\mathrm{e}\end{array}$ & $\left.\right|_{2 s, s y n} ^{\mathrm{H}}$ & $\underbrace{\mathrm{O} \text {, anti }}_{\mathrm{Cl}}$ \\
\hline R & Method & $t / \mathrm{h}$ & $\begin{array}{l}\text { Yields of } 2 \\
(\%)\end{array}$ & $2 \mathrm{~s}: 2 \mathrm{a}^{c}$ \\
\hline 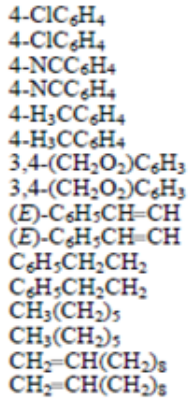 & $\begin{array}{l}\text { A } \\
\text { B } \\
\text { A } \\
\text { B } \\
\text { A } \\
\text { B } \\
\text { A } \\
\text { B } \\
\text { A } \\
\text { B } \\
\text { A } \\
\text { B } \\
\text { A } \\
\text { B } \\
\text { A } \\
\text { B }\end{array}$ & $\begin{array}{l}70 \\
71 \\
71 \\
68 \\
62 \\
72 \\
78 \\
97 \\
72 \\
96 \\
71 \\
96 \\
93 \\
95 \\
70 \\
94\end{array}$ & $\begin{array}{l}74 \\
80 \\
71 \\
72 \\
58 \\
49 \\
56 \\
24 \\
78 \\
53 \\
58 \\
64 \\
48 \\
48 \\
62 \\
63\end{array}$ & $\begin{array}{l}14: 86 \\
93: 7 \\
15: 85 \\
91: 9 \\
20: 80 \\
91: 9 \\
24: 76 \\
93: 7 \\
15: 85 a \\
77: 23^{\circ} \\
32: 68 \\
76: 24 \\
36: 64 \\
84: 16 \\
26: 74 \\
78: 22\end{array}$ \\
\hline
\end{tabular}

The allylation of aldehydes $(1.0 \mathrm{mmol})$ by $1(2.0 \mathrm{mmol})$ was carried out with $\mathrm{PdCl}_{2}\left(\mathrm{PhCN}_{2}(0.02 \mathrm{mmol}), \mathrm{SnX}_{2}(2.0 \mathrm{mmol}), \mathrm{TBAI}(0.2 \mathrm{mmol})\right.$ and $\mathrm{NaI}(2.0 \mathrm{mmol})$ in DMI $(3 \mathrm{ml})$ and $\mathrm{H}_{2} \mathrm{O}(0.1 \mathrm{ml})$. ${ }^{\circ}$ Isolated yields. 6 The ratio was determined by ${ }^{1} \mathrm{H}$ NMR spectroscopy (JEOL GX-270). $\$ d$ The ratios (cis: trans) refer to the corresponding vinyl epoxides.

Table 5. Reaction of allylic alcohol with aldehydes and some of the obtained results under exposure to ultrasonic irradiation [Adapted from Masuyama et al. (1994) Inorganica Chimica Acta 220. 155-159. Copyright (C) 1994, Elsevier].

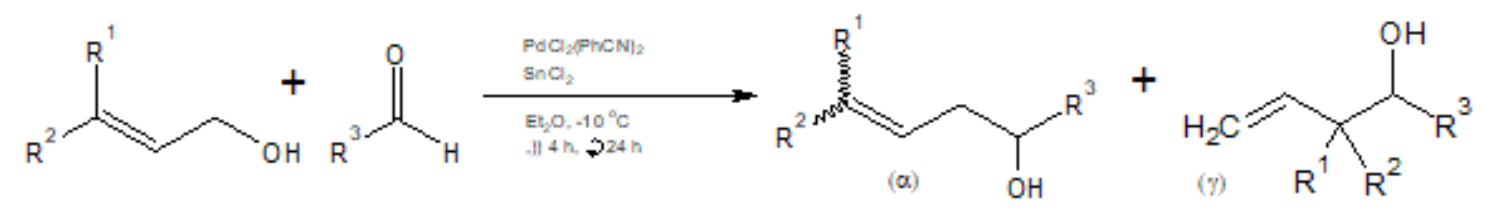

\begin{tabular}{|c|c|c|c|c|c|c|c|}
\hline \multirow{2}{*}{ Entry } & \multicolumn{2}{|l|}{ Alcohol } & \multirow{2}{*}{ Aldehyde $\mathrm{R}^{3}$} & \multirow{2}{*}{ Yield b $(\%)$} & \multicolumn{3}{|c|}{ Ratio " } \\
\hline & $\mathbb{R}^{1}$ & $\mathbb{R}^{2}$ & & & a.y & $E: Z$ & syn:anti \\
\hline 1 & $\mathrm{Me}$ & $\mathrm{H}$ & 4-MeOOCC $6 \mathrm{H}_{4}$ & 83 & $84: 16$ & $86: 14$ & $48: 52$ \\
\hline 2 & $\mathrm{Me}$ & $\mathrm{H}$ & 4- $\mathrm{NCC}_{6} \mathrm{H}_{4}$ & 51 & $69: 31$ & $85: 15$ & $56: 44$ \\
\hline 3 & $\mathrm{Me}$ & $\mathrm{H}$ & $4-\mathrm{ClC}_{6} \mathrm{H}_{4}$ & 49 & $66: 34$ & $93: 7$ & $57: 43$ \\
\hline 4 & $\mathrm{Me}$ & $\mathrm{H}$ & $\mathrm{c}-\mathrm{C}_{6} \mathrm{H}_{11}$ & 15 & $95: 5$ & $71: 29$ & $52: 48$ \\
\hline 5 & $\mathrm{Me}$ & $\mathrm{H}$ & $\mathrm{C}_{6} \mathrm{H}_{13}$ & 23 & $91: 9$ & $56: 44$ & $84: 16$ \\
\hline 6 & $\mathrm{CH}_{2}\left(\mathrm{CH}_{2}\right)_{2}$ & $\mathrm{H}$ & $\mathrm{C}_{6} \mathrm{H}_{5}$ & 57 & $98: 2$ & $67: 33$ & $70: 30$ \\
\hline 7 & $\mathrm{Me} 2 \mathrm{C}=\mathrm{CH}\left(\mathrm{CH}_{2}\right)_{2}$ & $\mathrm{Me}$ & $\mathrm{C}_{6} \mathrm{H}_{5}$ & 46 & $90: 10$ & $d$ & d \\
\hline 8 & $\mathrm{c}-\mathrm{C}_{6} \mathrm{H}_{11}$ & $\mathrm{H}$ & $\mathrm{C}_{6} \mathrm{H}_{5}$ & 69 & $99: 1$ & $99: 1$ & $d$ \\
\hline
\end{tabular}

Conditions: $\mathrm{PdCl}(\mathrm{PhCN}) 20.02 \mathrm{mmol}, 5 \mathrm{SCl} 25 \mathrm{mmol}$, allylic alcohol $3 \mathrm{mmol}$, ultrasonication $-10^{\circ} \mathrm{C}$, 4 h; aldehyde 1 mmol, $25^{\circ} \mathrm{C}, 24-48 \mathrm{~h}$. ${ }^{b}$ Isolated yields of the mixture of regioisomers based on aldehydes. The ratio was determined by ${ }^{1} \mathrm{H}$ NMR (GX-270) and or GC (capillary column PEG $20 \mathrm{M}, 0.25 \mathrm{~mm} \times 3 \mathrm{~m}$ ). ${ }^{d}$ The ratio of stereoisomers ( $E: Z$ or syn:anti) was not confirmed. 
Regioselective control is one of the key considerations of Masuyama's group in Pd-catalyzed carbonyl allylations. In 1994, he and his co-workers were able to publish their work on synthesizing $\alpha$-selected homoallylic alcohols using a $\mathrm{Pd}-\mathrm{SnCl}_{2}$ system under non-polar solvents (Masuyama, Hayakawa, Kurusu $\&$ Kishida, 1994a). With the low solubility of $\mathrm{SnCl}_{2}$ in non-polar solvents, the reaction was made to proceed by employing ultrasonic irradiation for 24$48 \mathrm{hrs}$. Results from their previous studies using polar solvents showed predominantly $\gamma$ products (Table 1) (Takahara, Masuyama \& Kurusu, 1992). The inverse was observed upon utilization of non-polar solvents, giving predominantly $\alpha$ regioisomers rather than the $\gamma$ counterpart. While this was the case, the method was only able to give moderate yields and generally low diastereoselectivity (Table 5).

\section{DEVELOPMENTS IN THE FIELD}

Much of the work highlighted in this paper are of Masuyama and his group, all involving the use of palladium catalysts and tin chloride for different carbonyl allylation reactions. The methods shown require long reaction periods and rather sophisticated chemicals to afford relatively moderate yields. Nevertheless, these works demonstrated the formation of new carbon-carbon bonds and the introduction of two functionalities (an alcohol and a double bond) with moderate to good regio- and diastereoselectivity. More recent developments in the field owe to Masuyama and associated authors their use of an umpolung of $\pi$-allylpalladium complexes in the presence of a low-valent metal as reducing agent. Some of these developments will be discussed in this section.

Use of Alternative Reagents. After Masuyama, many other works have stepped up to provide alternative or improved methods for carbonyl allylation. For example, in 2015, a highly diastereoselective allylation reaction of aldehydes and isatins with simple acyclic alkenes was developed (Tao, Li, Han \& Gong, 2015). This is reminiscent of the

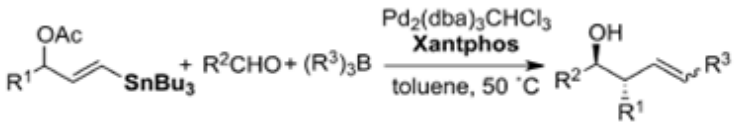

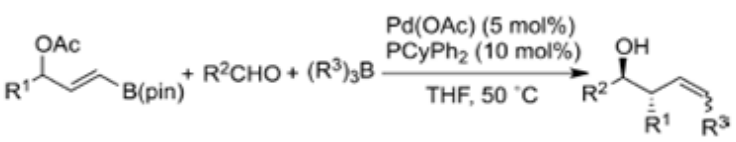

Scheme XI. Reaction system for the synthesis of (E)and (Z)-anti-homoallylic alcohol [Reprinted with permission from Horino et al. (2016) Chemical Communications. 1023-1028. Copyright (c) 2016, John Wiley \& Sons, Inc.].

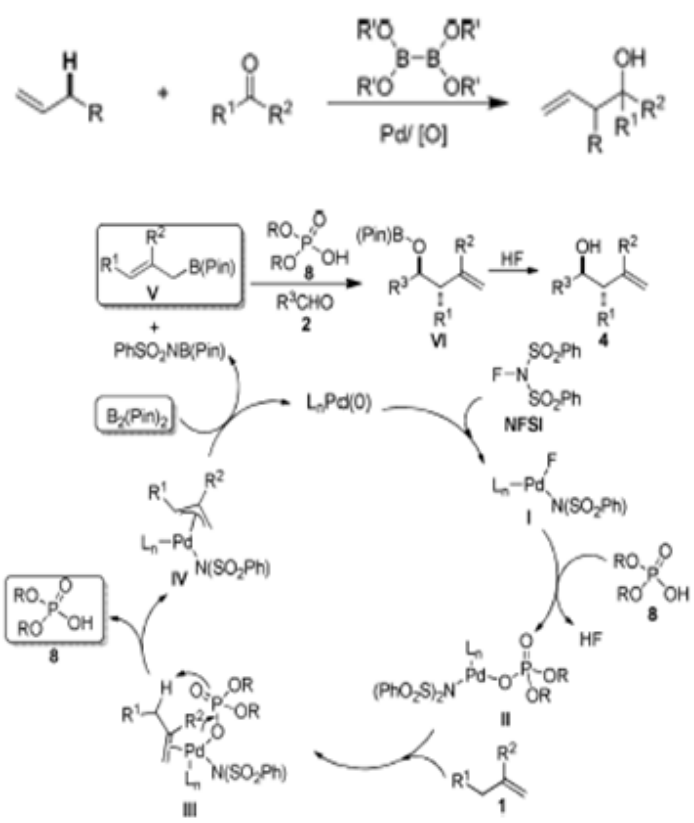

Scheme X. Proposed reaction pathway for the allylation of carbonyl compounds with simple olefins [Reprinted with permission from Tao et al. (2015) Journal of American Chemical Society. 137, 4054-4057. Copyright (C) 2015, American Chemical Society].

1994 work of Masuyama et al. involving carbonyl allylation using isoprene (Masuyama, Tsunoda \& Kurusu, 1994b). This method developed by Tao's group utilized a palladium catalyst, bis(pinacolato) diboron, an oxidant, and a Bronsted acid. The $50^{\circ} \mathrm{C}$-transformation was found to be a sequential process consisting of a $\mathrm{Pd}$-catalyzed oxidative allylic $\mathrm{C}-\mathrm{H}$ borylation and an allylboration of carbonyls accelerated by phosphoric acid. The oxidant involved, mainly N-fluorobenzenesulfonimide (NFSI), played a key role in the activation of the allylic $\mathrm{C}-\mathrm{H}$ while the Bronsted acid, phosphoric acid in this case, catalyzed the allylation reaction and facilitated the whole process (Scheme X). The study gave promising results, with moderate to excellent yields (64-99\%) and high diastereoselectivity, with $>20: 1$ anti:syn ratio. This was true for a broad range of substituted allylarenes having diverse steric and electronic properties, but those with neutral and cyclic allyl substituents were observed to give excellent yields. For aldehyde substrates, neither

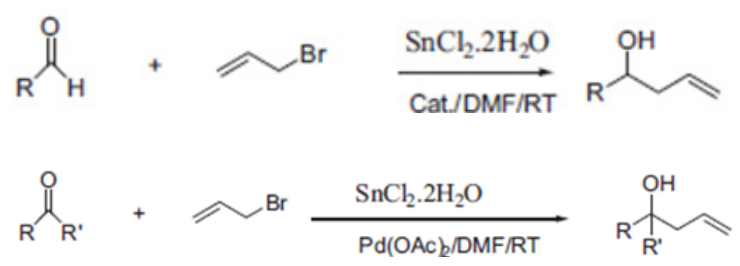

Scheme XII. Reaction system for the synthesis of allylic alcohols facilitated by $\mathrm{SnCl}_{2}-\mathrm{H}_{2} \mathrm{O}$ [Adapted from Kalita \& Phukan. (2013) Comptes Remdus Chimie. 1055-1062. Copyright (C) 2013, Elsevier]. 
Table 6. Reaction of allylic alcohol with aldehydes and some of the obtained results under exposure to ultrasonic irradiation [Adapted from Freitas $e t$ al. (2014). Ultrasonics Sonochemistry. Copyright (C) 2014, Elsevier].

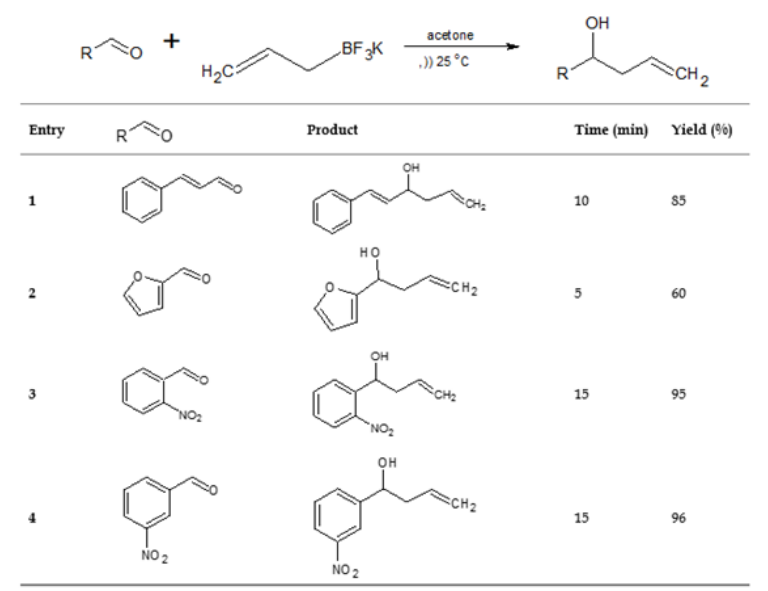

the electronic properties nor substitution patterns affected diastereoselectivity. Although this was the case, aldehydes with electron-deficient substituents gave higher yields compared to those of electron-rich substrates. Isatin derivatives were also experimented within the process and it was determined that both their electronic features and substitution patterns had little effect on diastereomeric ratios. Generally, a wide range of aldehydes and alkenes were found to be well tolerated.

2016 has also been a productive year in the field as yet another study for the facile process of $(E)$-antihomoallylic alcohol production was developed using a palladium-xantphos-catalyzed reaction system of 3 -(tri-n-butylstannyl)allyl acetates, aldehydes, and triorganoboranes (Scheme XI) (Horino, Sugata \& Abe, 2016). This served as alternative to the typical carbonyl allylation bound with the limitation of introducing $n$-alkyl, sec-alkyl, and aryl groups, which came from triorganoboranes, into the alkene. The supposed mechanism starts with the formation of an allylic gem-palladium/stannyl intermediate, which then undergoes allylation of the aldehyde via a coupling reaction of the in situ-generated $(E)$ vinylpalladium acetate with the triorganoboranes. The whole process took around $1 \mathrm{hr}$. at a moderate temperature of $50^{\circ} \mathrm{C}$, using toluene as solvent. Results were of moderate yields, particularly around 55$89 \%$, with $E / Z$ stereo- and diastereoselectivity ratios of around 5:1 - 13:1. Good to excellent yields were found for electron-neutral and electron-deficient aldehydes, heterocyclic aldehydes, heteroarylsubstituted substrates, and tri- $n$-alkylboranes. Aliphatic aldehydes however only gave moderate yields. Nevertheless, high diastereoselectivities were observed for all types of aldehydes and tri- $n$ -
Table 7. Reaction for the mechanochemical method of synthesizing homoallylic alcohols using allyltributylstannane, aldehyde, and phosphotungstic acid under solvent free conditions [Reprinted with permission from Bora et al. (2011) Canadian Journal of Chemistry. 90, 167-172. Copyright (C) 2011, NRC Research Press].

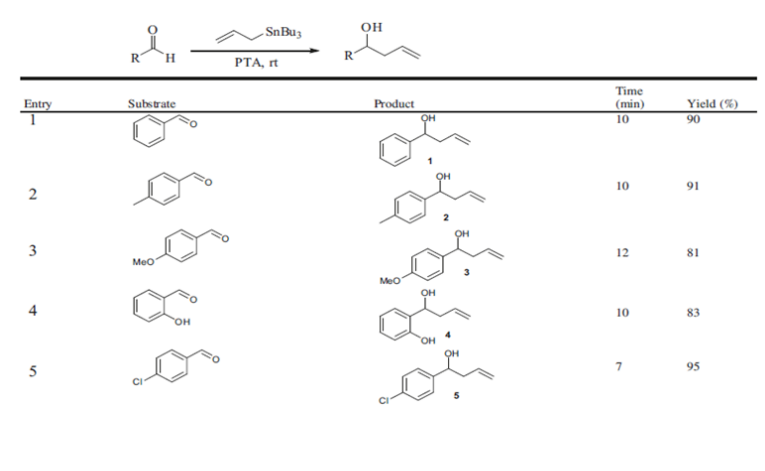

alkylboranes tested. Moderate diastereoselectivities were also observed for heteroaryl substrates. Previous studies of Horino and co-workers had also demonstrated the formation of $(Z)$-anti-homoallylic alcohol, but in this case, using 3-(pinacolatoboryl) allyl acetates, aldehydes, and triorganoboranes

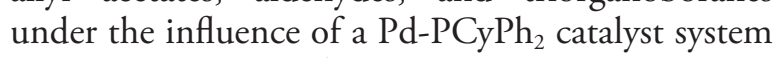
(Horino, Sugata \& Abe, 2016).

Both works of Tao et al. and Horino et al. provided alternative methods to that of Masuyamas. With the introduction of additional reagents to facilitate the process, significant increases in yield and extent of diastereoselectivity were observed. Reductions in reaction durations are also worth mentioning.

The presence of water has also been found to facilitate smooth palladium-catalyzed carbonyl allylation with allylic alcohol (Takahara, Masuyama \& Kurusu, 1992). A similar study utilized a palladium catalyst but in the presence of $\mathrm{SnCl}_{2}-\mathrm{H}_{2} \mathrm{O}$ under DMF solvent (Scheme XII) (Kalita \& Phukan, 2013). The study involved carbonyl allylation using allyl bromide. The results showed good yields (7593\%) even under short reaction periods: 1-3 hrs. for aldehydes and 5-8 hrs. for ketones. In general, the method worked well with aliphatic aldehydes to give corresponding products in excellent yields. Aromatic aldehydes bearing electron-withdrawing groups also reacted successfully, except for those bearing $-\mathrm{NO}_{2}$ and $-\mathrm{F}$ moieties, independent of the substituent position. This can be extended as well to a variety of ketones, giving appreciable yields. Most of the methods available for ketone allylation revealed that this $\mathrm{SnCl}_{2}$-mediated process needed long reaction times to achieve good yields ( $\mathrm{Li}$ et al., 1998). In the recent developments by Kalita and Phukan, it was 
Table 8. $\mathrm{Pd}^{0} / \mathrm{Ti}^{\mathrm{III}}$ (A) vs. $\mathrm{Ni}^{0} / \mathrm{Ti}^{\mathrm{III}}(\mathrm{B})$-catalyzed carbonyl allylations. Incomplete list from source [Reprinted with permission from MartinezPeragon et al. (2012) Eur. J. Org. Chem. 14991503. Copyright (c) 2012, John Wiley \& Sons, Inc.].

Entry $\begin{gathered}\text { Carbonyl } \\ \text { compound }\end{gathered}$
3-phenylpropanal

shown that ketone allylation can be completed in shorter reaction times, thus providing an alternative and efficient method for carbonyl allylation that employs almost just the same process. Although the study did not explore thoroughly on the stereoand regioselectivity of the process, it still provided good information on what conditions are favorable to achieve significant carbonyl allylation yields. Investigations on the regio- and stereoselectivity of this process could also be performed in the future to determine needed conditions.

Development of More Economic Methods. It has been of great interest to many organic chemists to develop synthetic methods that can be considered both "green" and efficient based on the use of less expensive and relatively easy to handle reagents. This section will now discuss alternative allylation methods and the use of relatively less toxic and more affordable chemical reagents for carbonyl allylation reactions. It was shown previously that Masuyama et al. were able to utilize non-polar solvents to synthesize $\alpha$-allylic carbonyl compounds using palladium catalysts and a tin chloride reductant (Masuyama, Hayakawa, Kishida \& Kurusu, 1994). The low solubility of $\mathrm{SnCl}_{2}$ in non-polar solvents was considered as an understandable barrier but was more or less remedied by the use of ultrasonic irradiation. For many years, ultrasonic irradiation has attracted considerable attention in organic
Table 9. Carboxylation of allylic acetates and allylic alcohols using Pd catalysts and $\mathrm{ZnEt}_{2}$ as reductant under $\mathrm{CO}_{2}$ atmosphere [Reprinted with permission from Mita et al. (2015) Chem. Eur. J. 21, 16391-16394. Copyright (c) 2015, John Wiley \& Sons, Inc.].

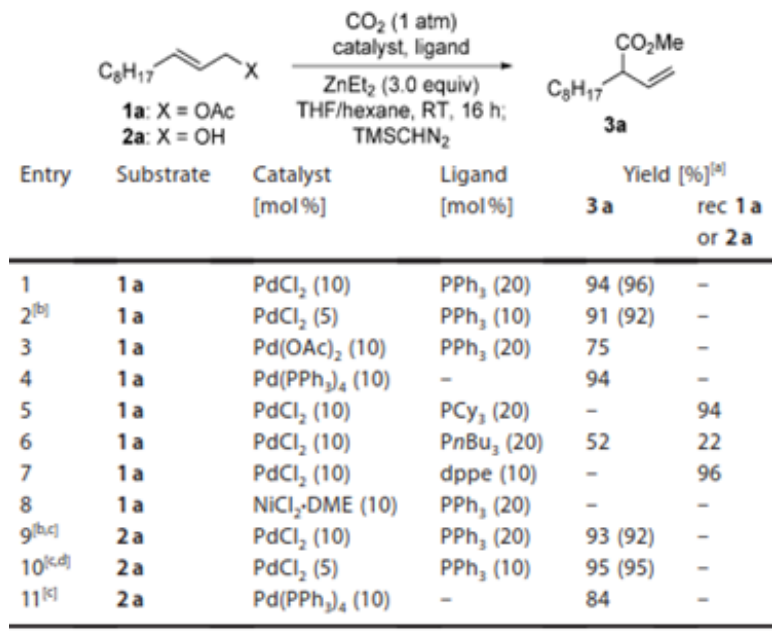

[a] Yields were determined by 'H NMR analysis using 1,1,2,2-tetrachloroethane as an internal standard. Isolated yields are given in parentheses. [b] Reaction time: $48 \mathrm{~h}$. [c] 3.5 equiv of $\mathrm{ZnEt}_{2}$ was used. [d] Reaction time: $72 \mathrm{~h} . \mathrm{ZnEt}_{2}: 1 \mathrm{M}$ solution in hexane.

synthesis mainly due to its ability to promote organic transformations that require drastic conditions and its ability to enhance reaction rates and increase yields. In 2014, Freitas and co-workers were able to develop an "environmentally benign reaction" for the synthesis of homoallylic alcohols based on the reaction of potassium allyltrifluoroborate and an aldehyde promoted by ultrasound. The method featured the use of small amounts of acetone as solvent without any additional metal catalysts or promoter (Table 6). Results have shown that aldehydes of aliphatic, aromatic, and heterocyclic types were efficiently allylated in moderate to high yields, $-60-96 \%$, in a quite short amount of time of only 5-30 minutes. Aromatic aldehydes containing electron-withdrawing and electron-donating groups gave corresponding products in high yields, but with the observation of much longer reaction periods with the latter. This was mainly due to electrostatic factors contributed by the high electron density on the carbonyl carbon. This served to react with another electron-rich source, an alkene. Additionally, substituent position was found to have no effect on the process as similar yields were observed for the test reactants. As for heterocyclic and aliphatic aldehydes, corresponding products were obtained in moderate yields. Freitas and co-workers attributed this to steric constraints and the loss of reactant due to volatilization, respectively. The regioselectivity and diastereoselectivity were also studied using 


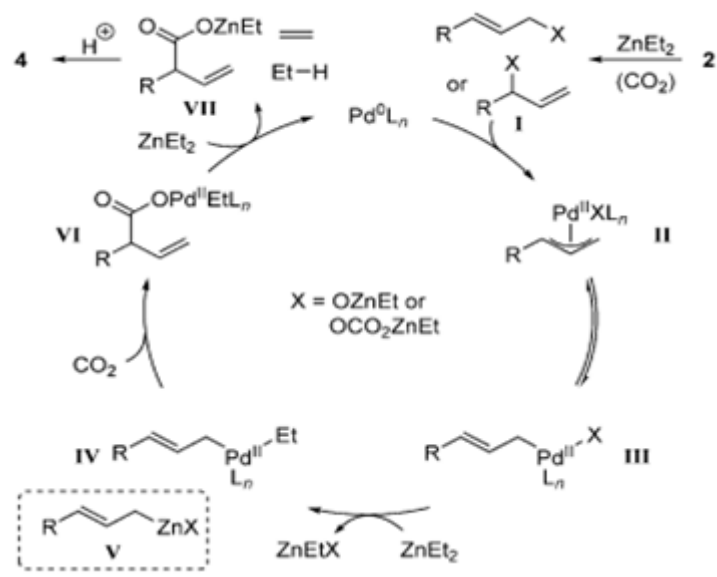

Scheme XIII. Proposed catalytic cycle for carboxylation of allylic substrates under $\mathrm{CO}_{2}$ atmosphere in the presence of $P d$ and $Z n E t_{2}$, where $2=$ allylic substrate and $4=$ carboxylated product [Reprinted with permission from Mita et al. (2015). Chem. Eur. J. 21, 16391-16394. Copyright (C) 2015, John Wiley \& Sons, Inc.].

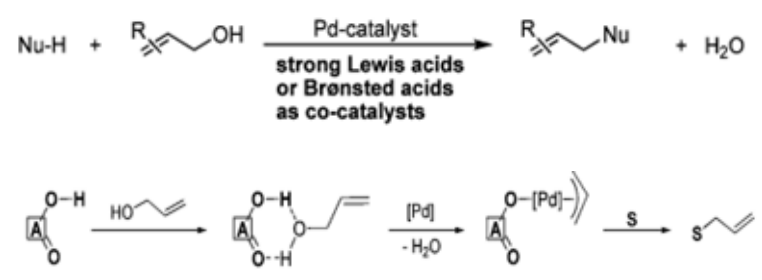

Scheme XIV. General reaction scheme and proposed mechanism for the activation of allylic alcohols using Bronsted acids [Reprinted with permission from Zhou et al. (2014) RSC Adv. 49, 25596-25599. Copyright (C) 2014, The Royal Society of Chemistry].

E-crotyltrifluoroborate and cinnamaldehyde and it was determined that the process can selectively give the $\gamma$-adduct in an 84:16 ratio (anti:syn). With these findings, the method of Freitas et al. can indeed be considered an efficient alternative to the early methods developed by Masuyama et al. (Freitas et al., 2014)

Another interesting method developed under carbonyl allylation employed mechanochemistry, whose main features used solvent-free conditions under the catalytic influence of environmentally benign phosphotungstic acid (PTA) (Bora, Sema, Wahlang \& Bez, 2011). The method also featured a tin derivative in the form of allyltributylstannane that reacts with an aldehyde upon grinding without solvent (Table 7). Aside from solvent free conditions, this method holds significant use for synthetic chemists as this provides easy handling, very short reaction times (5-20 mins), and aqueous free isolation protocols. Results are promising for both aliphatic and aromatic aldehydes, having excellent
Table 10. Tested reaction conditions for allylation of aldehydes with allylic alcohol and Pd/Bronsted acid catalyst system [Reprinted with permission from Jiang \& List. (2011). Adv. Synth. Catal. 353, 1667-1670. Copyright ( $)$ 2011, John Wiley \& Sons, Inc.].

$\begin{array}{llll}60 & \\ & & \\ \end{array}$

yields ranging from $81 \%$ to $99 \%$. The presence of electron-withdrawing and electron-donating groups on the phenyl rings also showed no direct effect on the compatibility of the method to yield the desired product. However, the work did not involve ketone allylation and diastereoselectivity investigations. Although this is so, this mechanochemical-type of reaction provides an intriguing alternative synthetic protocol for carbonyl allylation.

Use of Alternative Metals and Lewis Acids. Some novel ideas have also come up far after the work of Masuyama and of similar research. Interesting chemistry has been observed using similar reaction systems, but of different substrates and catalysts. For instance, a system using a nickel-based catalyst and a titanium-based reductant $\left(\mathrm{Ni}^{\circ} / \mathrm{Ti}^{\mathrm{III}}\right)$ showed good to excellent yields for carbonyl allylation using an allylic carbonate with a proposed mechanism highly reminiscent of the $\mathrm{Pd}^{0} / \mathrm{Sn}^{\mathrm{II}}$ system. Surprisingly, simple ketones and aromatic ketones also gave appreciable yields, similar with the use of palladium catalysts (Table 8). Reactions were even found to proceed at room temperature using catalytic amounts of the metal-based reagents (Martínezperagón et al., 2012). While palladium is evidently not unique in its ability to facilitate carbon-carbon bond forming reactions, being a noble metal makes it more practical to use, as palladium and its compounds are generally less susceptible to oxidation (Brooks, 1992). However, Martinez- 
Table 11. Carbonyl allylation from alcohol oxidation level via iridium-catalyzed coupling of allyl acetate and alcohols, using (R)-BINAP [Reprinted with permission from Kim et al. (2008). J. Am. Chem. Soc. 130, 6340-6341. Copyright ()$_{2008}$, American Chemical Society].

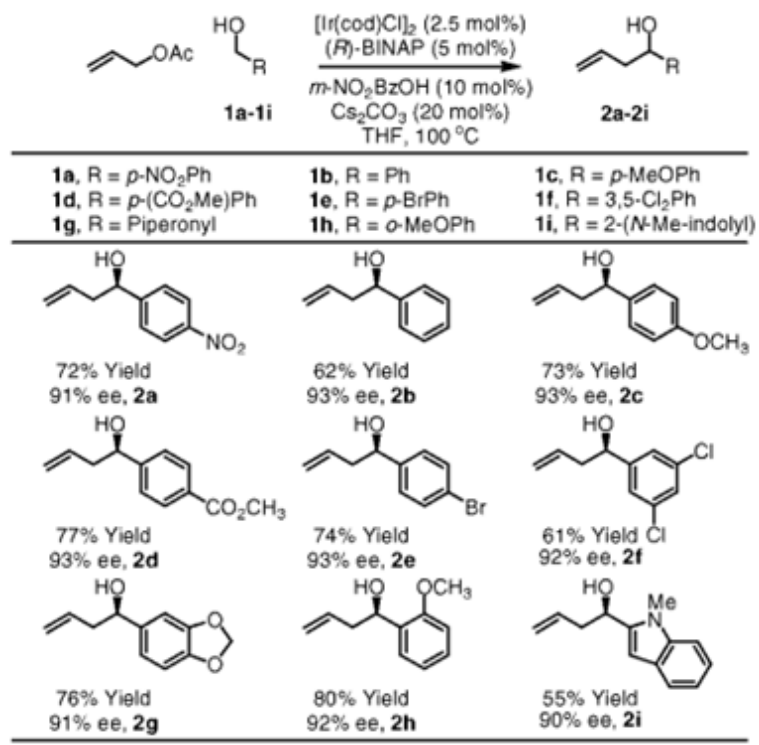

Peragon and her group have noted that, unlike the Pd-based system, their Ni-based reaction system did not promote allylation products when using substituted allylic carbonates, such as crotyl or prenyl derivatives, as pronucleophiles. This thus allowed the use of substrates containing such functionalities as the carbonyl compound or electrophile, making the nickel-based system more chemoselective than the palladium-based one. In addition, nickel-based catalysts are said to be more desirable in terms of cost as $\mathrm{Ni}$ is considered as a "commodity metal," whereas Pd and Pt are considered as precious metals, demanding higher prices (Rosen et al., 2011).

In another study, highly regioselective carboxylation of allylic alcohols was developed using merely $\mathrm{CO}_{2}$ as the electrophile (Mita, Higuchi \& Sato, 2015). In their simple but clever system, various palladium complexes were tested for efficiency, along with an appropriate ligand, $\mathrm{ZnEt}_{2}$ as reductant, and under $1 \mathrm{~atm}$ pressure of $\mathrm{CO}_{2}$ (Table 9). Their experiments found favorable yields of product for both allylic acetates and alcohols, and found that $\mathrm{PdCl}_{2}$ added with $\mathrm{PPh}_{3}$ ligand was generally the most favored catalyst system. The authors also found that the reaction regioselectively gave branched, instead of linear, carboxylic acid products in all cases and in high yields. Based on their data, as well as some previous reports, they have proposed a plausible catalytic cycle for their system as shown (Scheme XIII).
Table 12. Carbonyl allylation from aldehyde oxidation level via iridium-catalyzed coupling of allyl acetate and alcohols, using (-)-TMBTP and isopropanol [Reprinted with permission from Kim et al. (2008). J. Am. Chem. Soc. 130, 63406341. Copyright (C) 2008, American Chemical Society].

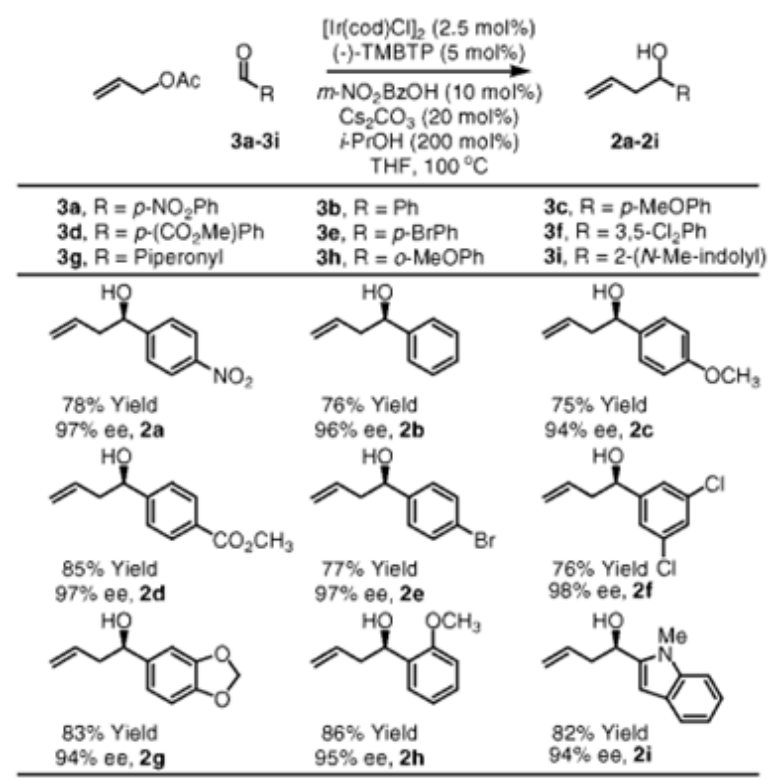

Another major development in the field is the replacement of Lewis acid activators with simpler Bronsted acids, such as carboxylic acids (Scheme XIV). Such a system is highly favored as it eliminates the production of metal-based waste (as in metalbased Lewis acids) and instead produces water and the recoverable conjugate base as by-products. Based off previous works of other authors, Jiang and List (2011) developed a system for the $\alpha$-allylation of aldehydes with allylic alcohols utilizing $\mathrm{Pd}\left(\mathrm{PPh}_{3}\right)_{4}$ as catalyst and either trifluoroacetic acid or benzoic acid as the Bronsted acid. They tested several reaction conditions for their system in search of an optimal one (Table 10). As suggested by their study, a reaction system of $\mathrm{Pd}\left(\mathrm{PPh}_{3}\right)_{4} / \mathrm{PhCO}_{2} \mathrm{H}$ in toluene solvent is the most effective with $>99 \%$ conversion. The reaction also exhibited a wide substrate scope, tolerating aromatics containing either an electronwithdrawing or electron-donating group, as well as aliphatic and unbranched aldehydes. Interestingly, another group has found that the reaction quite readily tolerates azlactone substrates without the need of a Bronsted acid as the substrate itself mediates the activation through its H-bonding moieties, allowing for economic syntheses of biologically active compounds such as quaternary allylic amino acids (Zhou et al., 2014).

Aside from using Bronsted acids, another approach that circumvents the stoichiometric use of metallic 

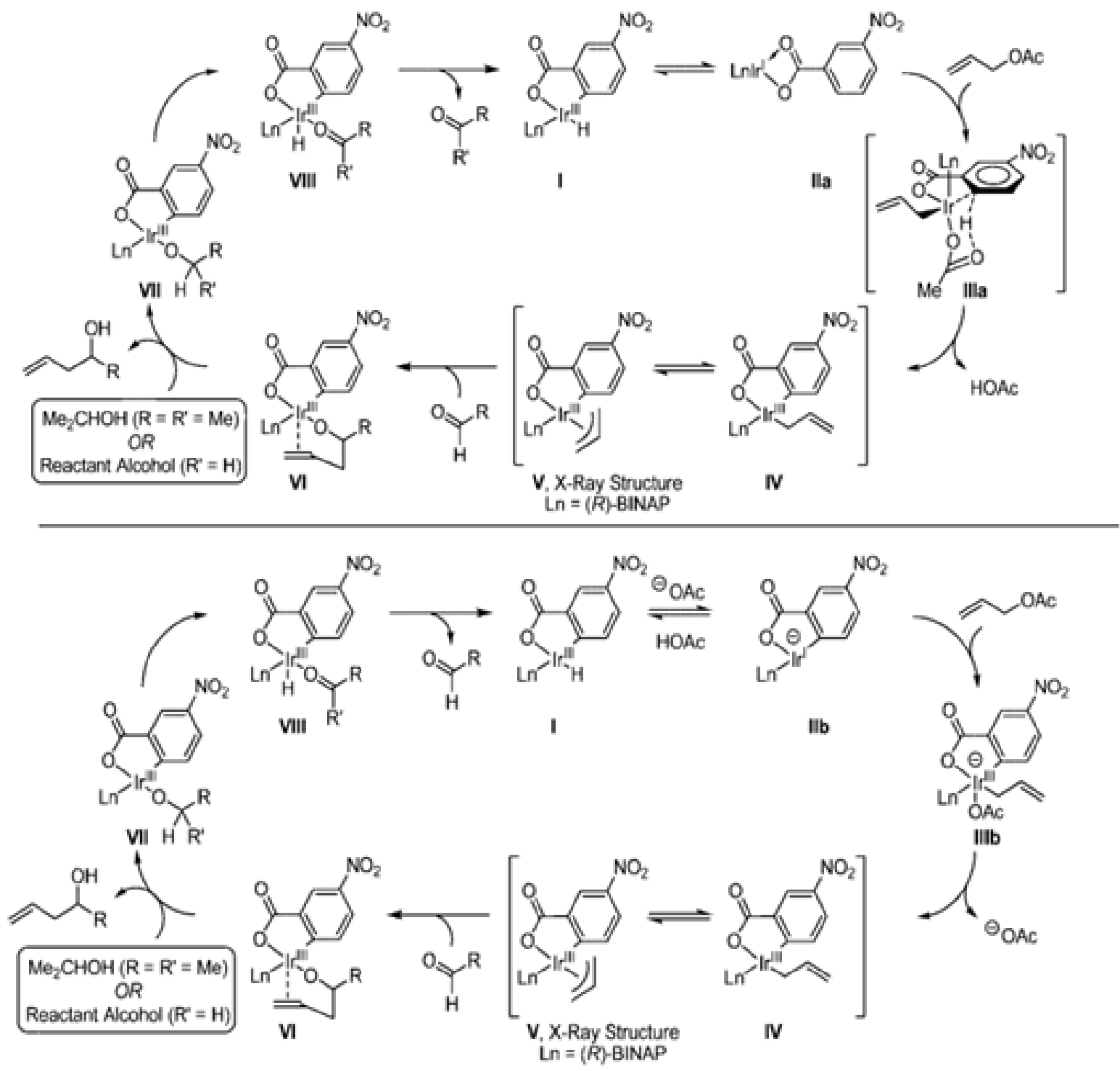

Scheme XV. Proposed catalytic mechanisms for the Ir-catalyzed transfer hydrogenative coupling from the aldehyde and or alcohol oxidation level ( $\mathrm{Ln}=$ chelating triaryl phosphne) [Reprinted with permission from Kim et al. (2008). J. Am. Chem. Soc. 130 (44), 14891-14899. Copyright (C) 2008, American Chemical Society].

reagents or Lewis acid activators is the utilization of transfer hydrogenative coupling. In this new family of catalytic carbonyl allylation methodologies, allenes, dienes, and allyl acetate can serve as precursors in transient allyl metal nucleophiles (Kim, Ngai \& Krische, 2008). From these three, allyl acetate has received much more attention due to its desirable allyl donor property, low cost, and tractability.

Remarkably, this approach does not only promote carbonyl allylation from the aldehyde oxidation level, but also from the alcohol oxidation level. Kim and co-workers (2008) reported an acetatealcohol transfer hydrogenative coupling in the presence of an iridium catalyst derived from $[\operatorname{Ir}(\operatorname{cod})$ $\mathrm{Cl}]_{2}$ and $(R)$-BINAP [cod=1,5-cyclooctadiene; BINAP $=(2,2 '-$ bis (diphenylphosphino $)-1,1$ 'binaphthyl)]. Products following C-type allylation were generated in good yields with exceptional enantioselectivity (Table 11). For allylation of aldehydes, nearly identical conditions were used except for employing isopropanol as the terminal reductant and (-)-TMBTP as the ligand (Table 12). Results from their preliminary analysis revealed that the utilization of $10 \mathrm{~mol} \% \mathrm{~m}$-nitrobenzoic acid additive and $20 \mathrm{~mol} \%$ cesium carbonate base gave the optimum results, and so were used for carrying out the reaction. This approach in carbonyl allylation was believed to happen based on a mechanism shown in Scheme XV (top), composed of the following steps: (1) formation of iridium carboxylate IIa from the association of $m-\mathrm{NO}_{2} \mathrm{BzOH}$ to $[\mathrm{Ir}(\operatorname{cod}) \mathrm{Cl}]_{2} ;$ (2) oxidative addition of allyl acetate to complex IIa; (3) acetate-assisted ortho-metalation through the six-centered transition structure IIIa to yield $\sigma$-allyl C,O-benzoate complex IV; (4) rapid equilibration of 
IV with the corresponding $\prod$-allyl haptomer $\mathrm{V}$; (5) allyl transfer to the aldehyde via chainlike transition structure to produce the homoallyl iridium alkoxide VI; (6) $\beta$-hydride elimination to deliver complex VIII; (7) and dissociation of aldehyde to regenerate the ortho-cyclometalated complex I.

Another related pathway was also deemed plausible, as shown by Scheme XV (bottom). The mechanism is almost identical with the previous one, and differs only in the first few parts of the process which comprise the (1) formation of anionic iridium (I) C,O-benzoate IIb from the ortho-cyclometalated complex I due to proton loss, and (2) oxidative addition of allyl acetate to provide the anionic iridium (II) $\sigma$-allyl complex IIIb, which upon loss of acetate then produces the neutral $\sigma$-allyl complexes IV and V, respectively. An Ir-based carbonyl allylation system thus shows promise for synthetic applications, as compared to Pd-based systems. In fact, studies have reported higher reaction yields when replacing the Pd-based catalyst with an Irbased one (Banerjee \& Roy, 2006; Masuyama \& Marukawa, 2007).

\section{CONCLUSIONS}

The efforts dedicated by Masuyama and co-workers in developing the methods for carbonyl allylation has indeed contributed significantly in the field of organic/organometallic synthesis and catalysis. Although the initial reaction systems produced only moderate yields despite long reaction durations and the use of quite toxic and rather costly reagents, these systems have served as the benchmark for future developments in the field. Carbonyl allylation has been dubbed as one of the most difficult organic transformations, and further developments in the reaction system, reagents and conditions to be used are still necessary to obtain an optimal system for the purposes of the chemist and his or her intended product. A special acknowledgement to Masuyama and co-workers should be given as their works have served as the foundation for the application of metalmediated charge reversal (umpolung) to carbonyl allylations in practical organic synthesis.

\section{ABOUT YOSHIRO MASUYAMA}

Professor Yoshiro Masuyama obtained his Bachelor in Engineering from Gunma University in 1973. He finished his Master's in Engineering and Doctor of Science in 1975 and 1978, respectively, from the Tokyo Institute of Technology. He was a research associate in the laboratory of Prof. K. Barry Trost at the University of Wisconsin at Madison from 1978 to 1980 . Thereafter, he taught and did research at Sophia University in Japan. He is involved in several works other than Pd-catalyzed and Sn(II)-mediated carbonyl allylation, namely: molybdenummediated/catalyzed reactions (Kurusu, Masuyama, Saito M \& Saito S, 1986; Masuyama, Takahashi \& Kurusu, 1984; Masuyama, Tsuhako \& Kurusu 1981; Masuyama, Kurusu \& Segawa, 1987a; Masuyama, Yamada \& Kurusu, 1987b; Trost \& Masuyama, 1984), tetrabromooxomolybdate complex-catalyzed oxidations (Kurusu \& Masuyama, 1986, 1985; Kurusu \& Masuyama, 1989; Kurusu \& Masuyama, 1989), synthesis of some dinuclear metal complexes (Guidote et al., 2001a, 2001b), hydrodesulfurization of dibenzothiophene derivatives over metal catalysts (Saih et al., 2005), various syntheses other than allylations using tin as catalyst, such as aziridination and amination (Masuyama, Ohtsuka, Harima \& Kurusu, 2006a), carbonyl propargylation (Masuyama, Yamazuki, Ohtsuka \& Kurusu, 2006c), aldol condensations (Masuyama, Ohtsuka \& Kondo, 2006b), cyclotrimerization of terminal alkynes (Masuyama, Miyazaki \& Suzuki, 2013a), and aldehyde-alkyne couplings (Masuyama, Takamura \& Suzuki, 2013b), synthesis and characterization of zirconium complexes (Podiyanachari et al., 2012; Suzuki et al., 2014, 2013, 2012, 2011b, 2011a) and even the synthesis of an amino acid used for diagnostic studies (Usuki et al., 2012).

\section{LITERATURE CITED}

Agenet N, Amatore C, Gamez S, Gérardin H, Jutand A, Meyer G, et al. Effect of the leaving group and the allylic structure on the kinetics and thermodynamics of the reaction of allylic carboxylates with palladium ( 0 ) complexes. ARKIVOC 2002;v:92-101.

Albéniz AC, Espinet P, Pérez-mateo A. Palladium (II) allylic complexes by carbene transmetalation and migratory insertion reactions: Synthesis and side reactions. J Organomet Chem 2010;695:4415. doi:10.1016/j.jorganchem.2009.10.027.

Amatore C, Gamez S, Jutand A, Meyer G, Morenoman M. Oxidative Addition of Allylic Carbonates to Palladium (0) Complexes: Chem - A Eur J 2000;6:3372-6.

Banerjee M, Roy S. Highly efficient Barbier allylation from allyl alcohol using iridium (I)/ tin (II): Unusual and indirect roles of allyl alcohol and tin. J Mol Catal A 2006;246:231-6. doi:10.1016/j. molcata.2005.11.009.

Bora PP, Sema HA, Wahlang B, Bez G. Rapid synthesis of homoallylic alcohol from aldehyde with allyltributylstannane under solvent-free conditions. Can J Chem 2011;90:167-72. doi:10.1139/v11138. 
Carrow BP, Hartwig JF. Distinguishing Between Pathways for Transmetallation in Suzuki-Miyaura Reactions. J Am Chem Soc 2011;133:2116-9. doi:10.1021/ja1108326.Distinguishing.

Caspi D. Palladium in Organic Synthesis. Top. Organomet. Chem., 2008, p. 1-13.

Crabtree R. The Organometallic Chemistry of the Transition Metals. New Jersey: John Wiley \& Sons, Inc.; 2005.

Denmark SE, Wilson T, Willson TM. On the Lewis-acid-induced addition of allylstannanes to aldehydes: a spectroscopic investigation. J Am Chem Soc 1988;110:984-6. doi:10.1021/ja00211a058.

Espinet P, Echavarren AM. The Mechanisms of the Stille Reaction. Angew Chemie Int Ed 2004;43:4704-34. doi:10.1002/anie.200300638.

Freitas JJR, Couto TR, Cavalcanti IH, Freitas JCR, Oliveira RA, Menezes PH. Metal free synthesis of homoallylic alcohols promoted by ultrasound. Ultrason Sonochem 2014;21:1609-14. doi:10.1016/j.ultsonch.2014.04.001.

Frost C, Howarth J, Williams J. Selectivity in Palladium Catalysed Allylic Substitution. Tetrahedron: Asymmetry 1992;3:1089-122.

Guidote, A.M. J, Ando K-I, Terada K, Kurusu Y, Nagao H, Masuyama Y. Synthesis, characterization and reactivity of a series of dinuclear copper complexes bearing the ligand bis[3-(2hydroxybenzylideneamino)phenyl] sulfone and derivatives. Inorganica Chim Acta 2001a;324:20311 .

Guidote AM, Ando K-I, Kurusu Y, Nagao H, Masuyama Y. Syntheses and characterization of homodinuclear manganese and cobalt complexes bridged by a hemiacetal or by an acetato group in a $\mu-(\eta 2: \eta 1)$ bridging mode. Inorganica Chim Acta 2001b;314:27-36.

Guimarães RL, Lima DJP, Barros MESB, Cavalcanti LN, Hallwass F, Navarro M, et al. Aqueous Barbier Allylation of Aldehydes Mediated by Tin. Molecules 2007;12:2089-105.

Hartings M. Reactions coupled to palladium 2012;4:2012. doi:10.1038/nchem.1437.

Horino Y, Sugata M, Abe H. Palladium-Catalyzed Three-Component Reaction of 3-(Tri-nbutylstannyl)allyl Acetates, Aldehydes, and Triorganoboranes: An Alternative to the Carbonyl
Allylation Using $\alpha, \gamma$-Substituted Allylic Tin Reagents. Adv Synth Catal 2016;358:1023-8. doi:10.1002/adsc.201501049.

Jiang G, List B. Palladium / Brønsted Acid-Catalyzed alpha-Allylation of Aldehydes with Allylic Alcohols. Adv Synth Catal 2011;353:1667-70. doi:10.1002/ adsc. 201100260.

Jutand A. Mechanism of Palladium-Catalyzed Reactions : Role of Chloride ions. Appl Organomet Chem 2004;18:574-82. doi:10.1002/aoc.742.

Kalita PK, Phukan P. SnCl2.2H2O-mediated Barbier-type allylation: A comparative evaluation of the catalytic performance of $\mathrm{CuI}$ and $\mathrm{Pd}(\mathrm{OAc}) 2$. Comptes Rendus Chim 2013;16:1055-62. doi:10.1016/j.crci.2013.02.012.

Kim IS, Ngai M, Krische MJ. Enantioselective Iridium Catalyzed Carbonyl Allylation from the Alcohol or Aldehyde Oxidation Level Using Allyl Acetate as an Allyl Metal Surrogate. J Am Chem Soc 2008;130:14891-9. doi:10.1021/ja802001b.

Kurosawa H, Emoto M, Kawasaki Y. Reductive elimination of hapta-3-allyl(aryl) palladium complexes promoted by allyl halides. J Organomet Chem 1988;346:137-46.

Kurusu Y, Masuyama Y. Oxidation of alcohols by hydroperoxides in the presence of polymersupported catalysts: Tetrabromooxomolybdate supported on a cationic polymer. J Macromol Sci Part A - Chem 1989;26:391-403.

Kurusu Y, Masuyama Y. Alcohol oxidation with t-butyl hydroperoxide in the presence of immobilized tetrabromooxomolybdate on silane coupling reagent-modified silica. J Polym Sci Part C, Polym Lett 1989;27:79-82.

Kurusu Y, Masuyama Y. Catalysis by tetrabromooxomolybdate $(V)$ complex: Oxidation of olefins and alcohols with t-butyl hydroperoxide. Polyhedron 1986;5:289-96.

Kurusu Y, Masuyama Y. Polymer-Supported Tetrabromooxomolybdate(V): A New Type of Catalyst for Oxidation by t-Butyl Hydroperoxide. J Macromol Sci 1985;24:389-401.

Kurusu Y, Masuyama Y, Saito M, Saito S. Epoxidation with t-butyl hydroperoxide in the presence of molybdenum peroxide and polymerimmobilized molybdenum peroxide. J Mol Catal 1986;37:235-41. 
Lei A, Srivastava M, Zhang X. Transmetalation of Palladium Enolate and Its Application in PalladiumCatalyzed Homocoupling of Alkynes: A RoomTemperature, Highly Efficient Route To Make Diynes. J Org Chem 2002;67:1969-71.

Lennox AJJ, Lloyd-Jones GC. Transmetalation in the Suzuki - Miyaura Coupling: The Fork in the Trail. Angew Chemie Int Ed 2013;52:7362-70. doi:10.1002/anie.201301737.

Li CJ, Meng Y, Yi XH, Ma J, Chan TH. Manganesemediated carbon-carbon bond formation in aqueous media: Chemoselective allylation and pinacol coupling of aryl aldehydes. J Org Chem 1998;63:7498-504. doi:10.1021/jo980535z.

Maitlis P. The Organic Chemistry of Palladium. Volume I. New York: Academic Press, Inc.; 1971.

Martínez-peragón Á, Millán A, Campaña AG, Rodríguez-márquez I, Resa S, Miguel D, et al. Ti I Ni-Based Multimetallic System for the Efficient Allylation of Carbonyl Compounds. European J Org Chem 2012:1499-503. doi:10.1002/ ejoc. 201101842 .

Masuyama Y. Palladium-catalyzed carbonyl allylation via pi-allylpalladium complexes. Adv. Met. Chem., vol. 3, JAI Press Inc.; 1994, p. 255-303.

Masuyama Y, Hayakawa A, Kurusu Y, Kishida M. Ultrasound-promoted carbonyl allylation allylic alcohols with tin ( II ) chloride in non-polar solvents. Inorganica Chim Acta 1994a;220:155-9.

Masuyama Y, Hayashi R, Takahara JP, Kurusu Y. Allylic Alcohols as Synthons of Allylic Carbanions. Palladium-Catalyzed Carbonyl Allylation by Allylic Alcohols with $\mathrm{SnCl}$. J Am Chem Soc 1988a; 110:4474-5.

Masuyama Y, Ito A, Kurusu Y. Either g-syn- or g-antiselective palladium-catalysed carbonyl allylation by mixed (E)- and (Z)-1,3-dichloropropene with tin (II) halides. Chem Commun 1998:315-6.

Masuyama Y, Kagawa M, Kurusu Y. Palladiumcatalysed carbonyl allylation by 2-methylenepropane1,3-diol. Chem Commun 1996:1585-6.

Masuyama Y, Kaneko Y, Kurusu Y. Rhodiumcatalyzed carbonyl allylations by allylic alcohols with tin (II) chloride. Tetrahedron Lett 2004;45:8969_ 71. doi:10.1016/j.tetlet.2004.10.051.

Masuyama Y, Kinugawa N, Kurusu Y. Charge Reversal of Electrophilic pi-Allylpalladium
Intermediates: Carbonyl Allylation by Allylic Acetates with $\mathrm{Pd}(\mathrm{PPh} 3) 4-\mathrm{Zn}$. J Org Chem 1987;52:3702-4.

Masuyama Y, Kurusu Y, Segawa K-I. Metal oxide-supported molybdenum carbonyl catalyst in liquid phase: effect of supports on regio- and stereoselectivity in allylic alkylation. J Mol Catal 1987a;40:183-93.

Masuyama Y, Marukawa M. Iridium-catalyzed carbonyl allylation by allyl ethers with tin (II) chloride. Tetrahedron Lett 2007;48:5963-5. doi:10.1016/j.tetlet.2007.06.117.

Masuyama Y, Miyazaki K, Suzuki N. Cyclotrimerization of Terminal Alkynes Catalyzed by a Phosphine-Free Chloro(1,5-cyclooctadiene) iridium(I) Dimer and Induced by Tin(II) Chloride. Asian J Org Chem 2013a;2:750-4.

Masuyama Y, Nimura Y, Kurusu Y. Palladiumcatalyzed carbonyl allylation by 2-(hydroxymethyl) acrylate derivatives: Synthesis of a-methylene-gbutyrolactones. Tetrahedron Lett 1991;32:225-8.

Masuyama Y, Ohtsuka M, Harima M, Kurusu Y. Tin(II) iodide-catalyzed selective aziridination or 1,2-diamination of alkenes with chloramine-T. Heterocycles 2006a;67:503-6.

Masuyama Y, Ohtsuka M, Kondo A. Aldol reactions of $\alpha$-bromoalkyl phenyl ketones and aldehydes with tin(IV) iodide and tetrabutylammonium iodide. Synlett 2006b:3346-8.

Masuyama Y, Otake K, Kurusu Y. Charge Reversal of Electrophilic pi-Allylpalladium Intermediates; Carbonyl Allylation by Allylic Acetates with $\mathrm{PdCl} 2(\mathrm{P}$ hCN)2-SnCl2. J Chem Soc Chem Commun 1988b:44-5.

Masuyama Y, Saeki K, Horiguchi S, Kurusu Y. A Novel Preparation of Allylic Trichlorotins from a a -Diisopropylhomoallylic Alcohols and Its Application to Carbonyl Allylations. Synlett 2001;11:1802-4.

MasuyamaY,Takahashi M,KurusuY.Chemoselective oxidation with molybdenum catalyst-t-butyl hydroperoxide. Tetrahedron Lett 1984;25:4417-20. doi:10.1016/S0040-4039(01)81454-4.

Masuyama Y, Takamura W, Suzuki N. Tin(II) chloride mediated coupling reactions between alkynes and aldehydes. European J Org Chem 2013b:8033-8. 
Masuyama Y, Takeuchi K, Kurusu Y. Carbonyl allylations by allylic chlorides utilizing a reduction of tin (IV) iodide to triiodostannate (II ) species with iodide sources. Tetrahedron Lett 2005;46:2861-3. doi:10.1016/j.tetlet.2005.02.113.

Masuyama Y, Tsuhako A, Kurusu Y. Dimethyl sulfoxide-catalytic molybdenum peroxide: A new system for the facile oxidation of alcohols. Tetrahedron Lett 1981;22:3973-6. doi:10.1016/ S0040-4039(01)82041-4.

Masuyama Y, Tsunoda M, Kurusu Y. Palladiumcatalysed Carbonyl Allylation by Isoprene. J Chem Soc Chem Commun 1994b:1451-2.

Masuyama Y, Yamada K, Kurusu Y. Molybdenum hexacarbonyl as a thiophilic metal reagent: desulfenylative allylation using allylic sulfides. 1Tetrahedron Lett 1987b;28:443-4.

Masuyama Y, Yamazuki R, Ohtsuka M, Kurusu Y. Carbonyl propargylation and allenylation with 2-propynyl mesylates, tin(IV) iodide, and tetrabutylammonium iodide controlled by either a steric effect or coordination effect. Synlett 2006c:1750-2.

Miessler GL, Fischer PJ, Tarr DA. Inorganic Chemistry. Harlow: Pearson Education Limited; 2014.

Mita T, Higuchi Y, Sato Y. Highly Regioselective Palladium-Catalyzed Carboxylation of Allylic Alcohols with CO2. Chem - A Eur J 2015;21:163914. doi:10.1002/chem.201503359.

Miyaura N. Cross-coupling reaction of organoboron compounds $\mathrm{v}$ ia base-assisted transmetalation to palladium (II) complexes. J Organomet Chem 2002;653:54-7.

Pearson R, Poulos A. Mechanism of Oxidative Addition of Allylic Halides to Iridium(I) Complexes. Inorganica Chim Acta 1979;34:67-76.

Podiyanachari SK, Fröhlich R, Daniliuc CG, Petersen JL, Kehr G, Erker G, et al. Facile formation of fivemembered N-heterocyclic zirconacycloallenoids. Dalt Trans 2012;41:10811-6.

Rosen BM, Quasdorf KW, Wilson DA, Zhang N, Resmerita AM, Garg NK, et al. Nickel-catalyzed cross-couplings involving carbon-oxygen bonds. Chem Rev 2011;111:1346-416. doi:10.1021/ cr100259t.

Roy AH, Hartwig JF. Directly Observed Reductive
Elimination of Aryl Halides from Monomeric Arylpalladium ( II ) Halide Complexes. J Am Chem Soc Commun 2003;125:13944-5.

Saih Y, Nagata M, Funamoto T, Masuyama Y, Segawa K. Ultra deep hydrodesulfurization of dibenzothiophene derivatives over $\mathrm{NiMo} / \mathrm{TiO} 2-$ Al2O3 catalysts. Appl Catal A Gen 2005;295:1122.

Sasaoka S, Yamamoto T, Kinoshita H, Inomata K, Kotake H. Palladium-Catalyzed Coupling of Allylic Acetates with Zinc. Chem Lett 1985:315-8.

Suzuki N, Nishimura K, Ohara N, Nishiura M, Masuyama Y. Studies on the mechanism for stereoisomerization of 1-zirconacyclopent-3- yne compounds. J Organomet Chem 2012;696:4321-6.

Suzuki N, Ohara N, Nishimura K, Sakaguchi Y, Nanbu S, Fukui S, et al. Characterization of the e isomer of tetrasubstituted [5]cumulene and trapping of the $\mathrm{Z}$ Isomer as a zirconocene complex. Organometallics 2011a;30:3544-8.

Suzuki N, Shimura T, Sakaguchi Y, Masuyama Y. Straightforward synthesis of five-membered metallacycloallenes:1-Zirconacyclopenta-2,3-diene compounds derived from 1,3-enynes. Pure Appl Chem 2011b;83:1781-8.

SuzukiN, Tsuchiya T, MasuyamaY. Synthesis ofsevenmembered cyclic allenes, 1-zirconacyclohepta-2,3,6trienes, from alkynes and 1-en-3-ynes. J Organomet Chem 2013;741-742:91-6.

Suzuki N, Yoshitani T, Inoue S, Hashizume D, Yoshida H, Tezuka M, et al. Haptotropic shift of [5] cumulenes in zirconocene complexes and effects of steric factors. Organometallics 2014;33:5220-30.

Takahara J, Masuyama Y, Kurusu Y. Palladiumcatalyzed carbonyl allylation by allylic alcohols with SnCl2. J Am Chem Soc 1992;1 14:2577-86.

Tan X, Hou Y, Liu L. SnCl2/Cu-Mediated Carbonyl Allylation Reaction in Water: Scope, Selectivity and Mechanism. Chinese J Chem 2004;22:450-4.

Tao ZL, LiXH, Han ZY, Gong LZ. Diastereoselective carbonyl allylation with simple olefins enabled by palladium complex-catalyzed $\mathrm{C}-\mathrm{H}$ oxidative borylation. J Am Chem Soc 2015;137:4054-7. doi:10.1021/jacs.5b00507.

Trost BM, Masuyama Y. Chemoselectivity in molybdenum catalyzed alcohol and aldehyde oxidations. Tetrahedron Lett 1984;25:173-6. 
Tsuiji J, Shimizu I, Minami I, Ohashi Y. Facile palladium-catalyzed decarboxylative allylation of active methylene compounds under neutral conditions using allylic carbonates. Tetrahedron Lett 1982;23:4809-12.

Tsuji J, Shimizu I, Minami I, Ohashi Y, Sugiura T, Takahashi K. Allylic Carbonates. Efficient Allylating Agents of Carbonucleophiles in Palladium-Catalyzed Reactions under Neutral Conditions. J Org Chem 1985:1523-9.

Usuki T, Yamada H, Hayashi T, Yanuma H, Koseki Y, Suzuki N, et al. Total synthesis of COPD biomarker desmosine that crosslinks elastin. Chem Commun 2012;48:3233-5.

Wang Z. Bariber reaction. Compr. Org. Name React. Reagents, John Wiley \& Sons, Inc.; 2010.

Zhou H, Yang H, Yin H, Liu M, Xia C, Jiang G. Palladium catalyzed direct allylation of azlactones with simple allylic alcohols in the absence of any. RSC Adv 2014;4:25596-9. doi:10.1039/ c4ra02477j. 